\title{
A Novel Four-Wing Hyperchaotic Complex System and Its Complex Modified Hybrid Projective Synchronization with Different Dimensions
}

\author{
Jian Liu, ${ }^{1,2}$ Shutang Liu, ${ }^{1}$ and Fangfang Zhang ${ }^{1}$ \\ ${ }^{1}$ School of Control Science and Engineering, Shandong University, Jinan 250061, China \\ ${ }^{2}$ School of Mathematical Sciences, University of Jinan, Jinan 250022, China \\ Correspondence should be addressed to Jian Liu; ss_liuj@ujn.edu.cn
}

Received 4 March 2014; Revised 12 May 2014; Accepted 16 May 2014; Published 5 June 2014

Academic Editor: Shurong Sun

Copyright (C) 2014 Jian Liu et al. This is an open access article distributed under the Creative Commons Attribution License, which permits unrestricted use, distribution, and reproduction in any medium, provided the original work is properly cited.

\begin{abstract}
We introduce a new Dadras system with complex variables which can exhibit both four-wing hyperchaotic and chaotic attractors. Some dynamic properties of the system have been described including Lyapunov exponents, fractal dimensions, and Poincaré maps. More importantly, we focus on a new type of synchronization method of modified hybrid project synchronization with complex transformation matrix (CMHPS) for different dimensional hyperchaotic and chaotic complex systems with complex parameters, where the drive and response systems can be asymptotically synchronized up to a desired complex transformation matrix, not a diagonal matrix. Furthermore, CMHPS between the novel hyperchaotic Dadras complex system and other two different dimensional complex chaotic systems is provided as an example to discuss increased order synchronization and reduced order synchronization, respectively. Numerical results verify the feasibility and effectiveness of the presented schemes.
\end{abstract}

\section{Introduction}

The real Lorenz system is a very simplified model of thermal convection in fluids (the Rayleigh-Benard problem) as

$$
\begin{aligned}
& \dot{x}=\sigma(y-x), \\
& \dot{y}=r_{1} x-x z-y, \\
& \dot{z}=x y-b z,
\end{aligned}
$$

where $x, y$, and $z$ are real state variables, $\sigma>0, r_{1}>0, b>0$ are real parameters, and a dot denotes a derivative with respect to time [1,2]. Later on, problems of laser physics [3] and of so-called baroclinic instability of the geophysical flows (in the atmosphere or in the ocean) unexpectedly resulted in a system of the same structure as (1) but in the complex domain $[4,5]$

$$
\begin{aligned}
& \dot{x}=\sigma(y-x), \\
& \dot{y}=r x-x z-a y, \\
& \dot{z}=\frac{1}{2}(\bar{x} y+x \bar{y})-b z,
\end{aligned}
$$

where the Rayleigh number $r$ and parameter $a$ are complex numbers defined by $r=r_{1}+j r_{2}$ and $j=\sqrt{-1}$ is the imaginary unit, and $\sigma, b, r_{1}, r_{2}, \delta$ are real and positive. The complex variables $x$ and $y$ and real variable $z$ of system (2) are related, respectively, to electric field and the atomic polarization amplitudes and the population inversion in a ring laser system of two-level atoms; an overbar denotes complex conjugate variable, chaotic motion of system (2) in Figure 1. In 2007, Mahmoud et al. [6] studied basic properties and chaotic synchronization of the complex Lorenz model as follows:

$$
\begin{aligned}
& \dot{x}=\sigma(y-x), \\
& \dot{y}=r_{1} x-x z-y, \\
& \dot{z}=\frac{1}{2}(\bar{x} y+x \bar{y})-b z,
\end{aligned}
$$

where $x, y$ are complex state variables, $z$ is real state variable, and $\sigma>0, r_{1}>0, b>0$ are real parameters. The complex Lorenz model (3) is embedded in system (2) and can be recovered when $r=r_{1}$ and $a=1$; that is, $r_{2}=\delta=0$. 


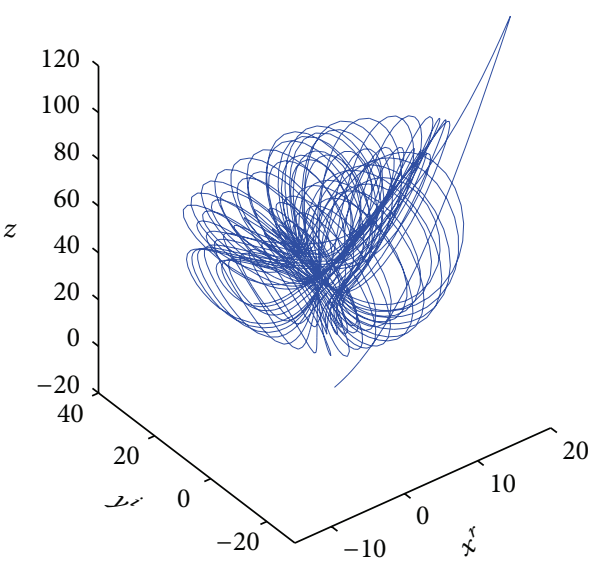

(a) on $\left(x^{r}, y^{i}, z\right)$ space

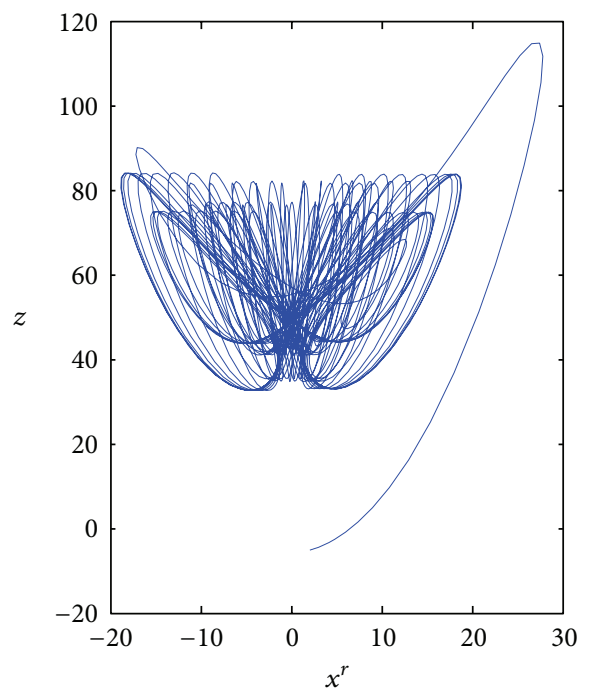

(c) on $\left(x^{r}, z\right)$ plane

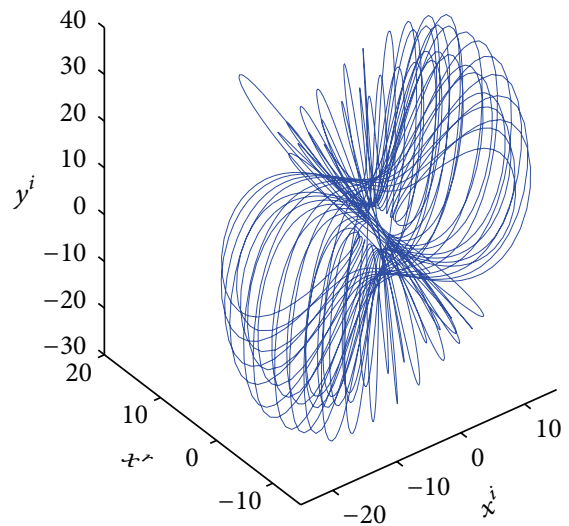

(b) on $\left(x^{i}, x^{r}, y^{i}\right)$ space

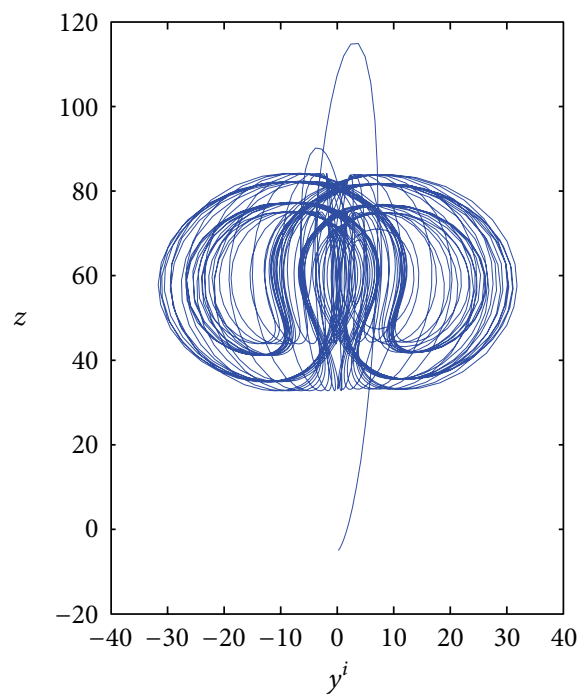

(d) on $\left(y^{i}, z\right)$ plane

FIGURE 1: The attractors of complex chaotic Lorenz system (2) with complex parameters $\sigma=5, r=60+0.2 j, a=1-0.6 j$, and $b=1.8$ and initial values $x(0)=2+0.2 j, y(0)=15+0.2 j$, and $z(0)=-5$.

In recent years, several other such examples have been proposed, notably the so-called complex Chen, Lü systems [7], and so on. Actually, many systems which involve complex variables have played an important role in many areas, including loading of beams and plates [8], optical systems [9], plasma physics [10], rotor dynamics [11], and high-energy accelerators [12]. Theoretical studies have focused on finding approximate solutions to various classes of complex valued equations $[13,14]$ and on dynamics and control of chaos $[15,16]$.

Due to the above wide scope of applications, many researchers devoted much effort to chaotic (hyperchaotic) synchronization as well. The global synchronization of coupled identical systems is well investigated in [7]. G. M. Mahmoud and E. E. Mahmoud [17] introduced the phenomenon of projective synchronization (PS) and modified projective synchronization (MPS) of hyperchaotic attractors of hyperchaotic complex Lorenz system by active control. PS is a situation in which the state variables of the drive and response systems synchronize up to a real scaling factor $\alpha$ ( $\alpha$ is a constant). MPS is defined if the responses of the synchronized dynamical states synchronize up to a real constant scaling matrix. Later on, P. Liu and S. Liu [18] presented full state hybrid projective synchronization (FSHPS) with real scaling factors for two complex chaotic systems according to the definition of FSHPS for real chaotic systems [19, 20]. Zhang and Liu [21] dealt with full state hybrid projective synchronization (CFSHPS) with complex scaling factors in 2014. Zhang et al. [22] discussed CMPS with complex scaling factors of uncertain real chaos and complex chaos in 2013 and G. M. Mahmoud and E. E. Mahmoud [23] achieved CMPS which can be synchronized with complex scaling matrix of two chaotic complex systems in 2013.

However, the aforementioned papers only consider chaotic synchronization of the same dimensional chaotic systems with complex variable, and the states of the drive and response systems projective synchronize up to a diagonal matrix, so each state variable of drive system is synchronized with a single state variable in response system up to a special scaling factor. In fact, the synchronization can be carried 
out through the oscillators with different dimensions and different structure, especially the systems in communication $[24,25]$, biological science, and social science [26], where the drive and response systems could be asymptotically synchronized up to a desired transformation matrix, not a diagonal matrix. By means of state transformation, multiple state variables in response system with respective scaling factor will be involved for a corresponding state variable of drive system. In applications to secure communication, Mahmoud et al. had dealt with PS of hyperchaotic complex nonlinear systems and proposed a corresponding communication scheme [27]. PS has been especially extensively studied because it can be used to obtain faster communication with its proportional feature and the unpredictability of the scaling factor can additionally enhance the security of communication. It is obvious that complex transformation matrix is arbitrary and more unpredictable than scaling matrix. Hence, the CMHPS with different dimensions will increase the complexity of the synchronization and further increase the diversity and the security of communications.

Moreover, to the best of our knowledge, almost all of the papers were devoted to synchronization of the complex systems with real parameters such as system (3), which are extreme cases of chaotic synchronization of the complex systems with complex parameters such as system (2) if all of the imaginary parts of complex parameters are equal to zero, and CMHPS between different dimensional hyperchaotic and chaotic complex systems with complex parameters, such as system (2), has rarely been explored. Therefore, it is meaningful and valuable to propose a general method to achieve CMHPS in chaotic complex systems with complex parameters.

Motivated by the above discussions, this paper introduces a new complex Dadras system which can exhibit both fourwing hyperchaotic and chaotic attractors and propose a new type of synchronization method of CMHPS for hyperchaotic and chaotic complex systems with complex parameters and with different dimensions, where the drive and response systems could be asymptotically synchronized up to a desired complex transformation matrix, not a diagonal matrix. In the complex space, the response system synchronizes with the projection of the drive system in a systematic way; that is to say, all the states of the response system are successfully synchronous in some sense with the states of the drive system. In addition, as a generalization of synchronization, depending on the form of the transformation matrix, CMHPS will contain PS, MPS, FSHPS, CPS, CMPS, and CFSHPS and extend previous works.

The rest of this paper is organized as follows. Section 2 introduces a new complex Dadras system which can exhibit both four-wing hyperchaotic and chaotic attractors. Some dynamic properties of the system have been described including Lyapunov exponents, fractal dimensions, and Poincaré maps. In Section 3, we address a new type of synchronization scheme of CMHPS for hyperchaotic and chaotic complex systems with complex parameters and with different dimensions, and two examples are presented to illustrate the effectiveness of the proposed scheme. Finally, conclusion is drawn in Section 4.
Notation. $\mathbb{C}^{n}$ denotes $n$ dimensional complex vector space and $\mathbb{C}^{m \times n}$ stands for $m \times n$ complex matrix space. If $z \in \mathbb{C}^{n}$ is a complex vector, then $z=z^{r}+j z^{i}, j=\sqrt{-1}$ is the imaginary unit, superscripts $r$ and $i$ stand for the real and imaginary parts of $z$, respectively, $z^{H}, z^{T}$ are the conjugate transpose and transpose of $z$, respectively, and $\|z\|$ implies the $L_{2}$-norm of $z$. If $z$ is a complex scalar, $|z|$ indicates the modulus of $z$ and $\bar{z}$ is the conjugate of $z$ and a dot denotes a derivative with respect to time, while $D^{H}$ is the conjugate transpose of $D$, provided that $D$ is a complex matrix.

\section{A New Hyperchaotic Complex Dynamic System}

2.1. The Proposed Complex Dynamic System. In 2012, Dadras et al. constructed a new real 4D smooth autonomous hyperchaotic nonlinear system [28], which is briefly referred to as the Dadras system as follows:

$$
\begin{aligned}
& \dot{x}=\alpha x-y z+w, \\
& \dot{y}=x z-\beta y, \\
& \dot{z}=x y+x w-\gamma z, \\
& \dot{w}=-y,
\end{aligned}
$$

where $(x, y, z, w)^{T}$ is the real state vector and $\alpha, \beta$, and $\gamma$ are positive constant parameters. System (4) can generate a fourwing hyperchaotic attractor when $\alpha=8, \beta=40$, and $\gamma=14.9$ in Figure 2 and a four-wing chaotic attractor when $\alpha=8$, $\beta=40$, and $\gamma=49$. In this paper, we discuss the complex extension of the Dadras system (4), which is expressed by

$$
\begin{aligned}
& \dot{z}_{1}=a z_{1}-z_{2} z_{3}+z_{4}, \\
& \dot{z}_{2}=z_{1} z_{3}-b z_{2}, \\
& \dot{z}_{3}=\frac{1}{2}\left[\bar{z}_{1}\left(z_{2}+z_{4}\right)+z_{1}\left(\bar{z}_{2}+\bar{z}_{4}\right)\right]-c z_{3}, \\
& \dot{z}_{4}=-\frac{1}{2}\left(z_{2}+\bar{z}_{2}\right),
\end{aligned}
$$

where $a, b$, and $c$ are real constant parameters, $z_{1}=u_{1}+j u_{2}$ and $z_{2}=u_{3}+j u_{4}$ are complex state variables, and $z_{3}=u_{5}$ and $z_{4}=u_{6}$ are real state variables. The complex Dadras system (5) can be rewritten as a real first-order ordinary differential equation (ODE) of the form

$$
\begin{aligned}
& \dot{u}_{1}=a u_{1}-u_{3} u_{5}+u_{6}, \\
& \dot{u}_{2}=a u_{2}-u_{4} u_{5}, \\
& \dot{u}_{3}=u_{1} u_{5}-b u_{3}, \\
& \dot{u}_{4}=u_{2} u_{5}-b u_{4}, \\
& \dot{u}_{5}=u_{1}\left(u_{3}+u_{6}\right)+u_{2} u_{4}-c u_{5}, \\
& \dot{u}_{6}=-u_{3} .
\end{aligned}
$$

2.2. Dynamic Properties of the New System. System (6) has the following dynamic properties. 


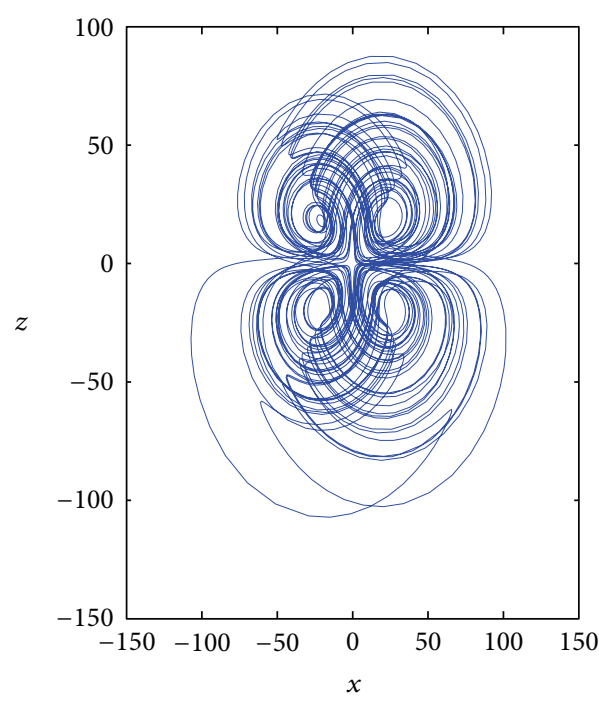

(a) on $(x, z)$ plane

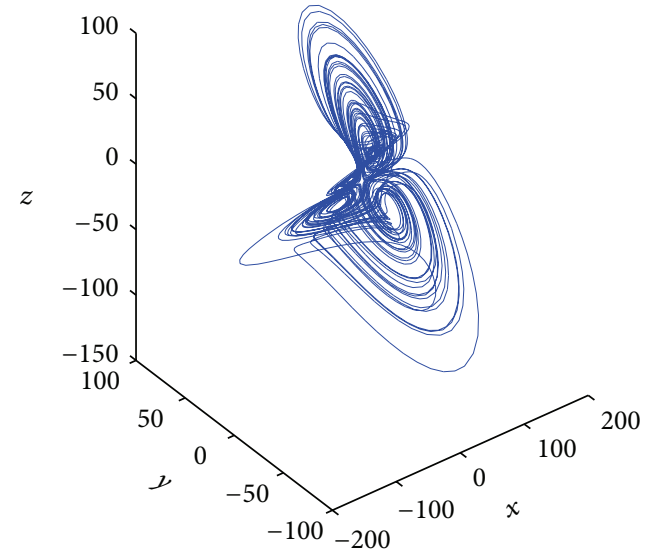

(b) on $(x, y, z)$ space

FIgURE 2: The four-wing hyperchaotic attractors of real Dadras system (4) for $\alpha=8, \beta=40$, and $\gamma=14.9$.

2.2.1. Symmetry and Invariance. System (6) is symmetrical about the $u_{5}$-axis, due to its invariance under the coordinates transformation $\left(u_{1}, u_{2}, u_{3}, u_{4}, u_{5}, u_{6}\right) \rightarrow\left(-u_{1},-u_{2},-u_{3},-u_{4}\right.$, $\left.u_{5},-u_{6}\right)$. In particular, the symmetry about the $u_{5}$-axis for any choice of parameters $a, b$, and $c$ is accurate.

2.2.2. Dissipation and the Existence of Attractor. For system (6), one has

$$
\nabla V=\sum_{k=1}^{6} \frac{\partial \dot{u}_{k}}{\partial u_{k}}=2 a-2 b-c .
$$

Hence, in order to ensure that system (6) is dissipative, it is required that $2 a-2 b-c<0$. Under this condition, system (6) converges exponentially:

$$
\frac{d V}{d t}=(2 a-2 b-c) V \Longrightarrow V=V_{0} e^{(2 a-2 b-c) t},
$$

$$
J=\left[\begin{array}{cccccc}
a & 0 & -u_{5} & 0 & -u_{3} & 1 \\
0 & a & 0 & -u_{5} & -u_{4} & 0 \\
u_{5} & 0 & -b & 0 & u_{1} & 0 \\
0 & u_{5} & 0 & -b & u_{2} & 0 \\
u_{3}+u_{6} & u_{4} & u_{1} & u_{2} & -c & u_{1} \\
0 & 0 & -1 & 0 & 0 & 0
\end{array}\right] \stackrel{E_{1}=(0,0,0,0,0,0)}{\longrightarrow} J_{E_{1}}=\left[\begin{array}{cccccc}
a & 0 & 0 & 0 & 0 & 1 \\
0 & a & 0 & 0 & 0 & 0 \\
0 & 0 & -b & 0 & 0 & 0 \\
0 & 0 & 0 & -b & 0 & 0 \\
0 & 0 & 0 & 0 & -c & 0 \\
0 & 0 & -1 & 0 & 0 & 0
\end{array}\right] .
$$

So, the eigenvalues of the Jacobian matrix $J_{E_{1}}$ are obtained as follows:

$$
\begin{aligned}
& \left|\mu I-J_{E_{1}}\right|=0 \Longrightarrow \mu_{1}=\mu_{2}=a, \\
& \mu_{3}=0, \quad \mu_{4}=\mu_{5}=-b, \quad \mu_{6}=-c .
\end{aligned}
$$

where the initial volume element is $V_{0}$. This implies that each volume containing the system trajectory shrinks to zero as $t \rightarrow \infty$ at an exponential rate, $2 a-2 b-c$. In fact, numerical simulations have shown that system orbits are ultimately confined into a specific limit set of zero volume, and the system asymptotic motion settles onto an attractor.

2.2.3. Equilibria and Stability. The equilibria of system (6) can be found by solving the following algebraic equations simultaneously: $a u_{1}-u_{3} u_{5}+u_{6}=0, a u_{2}-u_{4} u_{5}=0, u_{1} u_{5}-$ $b u_{3}=0, u_{2} u_{5}-b u_{4}=0, u_{1}\left(u_{3}+u_{6}\right)+u_{2} u_{4}-c u_{5}=0,-u_{3}=0$. Hence, one has five isolated fixed equilibria: $E_{1}=$ $(0,0,0,0,0,0), E_{2,3}=( \pm \sqrt{a c}, \pm \sqrt{a b}, 0,0, \sqrt{b c}, 0)$, and $E_{4,5}=$ $( \pm \sqrt{a c}, \mp \sqrt{a b}, 0,0,-\sqrt{b c}, 0)$. Here, the stability of the zero equilibrium $E_{1}$ is discussed. The Jacobian matrix of system (6) is

Since $a, b$, and $c$ are all positive real numbers, one can easily find $\mu_{1}=\mu_{2}>0, \mu_{3}=0, \mu_{4}=\mu_{5}<0, \mu_{6}<0$, implying that the equilibrium $E_{1}$ is unstable.

2.2.4. Lyapunov Exponents and Fractal Dimensions. In this section, the numerical simulations are carried out using 
MATLAB program. The fourth order Runge-Kutta integration algorithm is performed to solve the differential equations. The initial condition is $x_{0}=(10+j, 10+j, 10,1)^{T}$ and system (6) can display four-wing hyperchaotic and four-wing chaotic attractors.

(i) Four-Wing Hyperchaotic Attractor. Setting the parameters as $a=10, b=40$, and $c=14.9$, the system (6) is hyperchaotic and can generate a four-wing hyperchaotic attractor. So, complex Dadras system (5) can display fourwing hyperchaotic attractor which is shown in Figure 3. The Lyapunov exponents [29] have been calculated as

$$
\begin{aligned}
& L E_{1}=2.80065, \quad L E_{2}=0.703862, \\
& L E_{3}=0, \quad L E_{4}=-0.184465, \\
& L E_{5}=-29.7874, \quad L E_{6}=-48.4024 .
\end{aligned}
$$

And the fractal dimension is

$$
\begin{aligned}
D_{L} & =j+\frac{1}{\left|L E_{j+1}\right|} \sum_{i=1}^{j} L E_{i} \\
& =4+\frac{L E_{1}+L E_{2}+L E_{3}+L E_{4}}{\left|L E_{5}\right|}=4.11146 .
\end{aligned}
$$

Both Lyapunov exponents and fractal dimension indicate that the system (5) is hyperchaotic with the aforementioned set of parameters.

(ii) Four-Wing Chaotic Attractor. Setting the parameters as $a=$ $10, b=40$, and $c=49$, the system (6) has generated a fourwing chaotic attractor. So, complex Dadras system (5) can display four-wing chaotic attractor in Figure 4. The Lyapunov exponents of the system in this case are

$$
\begin{aligned}
& L E_{1}=0.915193, \quad L E_{2}=0, \\
& L E_{3}=-0.0910834, \quad L E_{4}=-2.8148, \\
& L E_{5}=-30.0099, \quad L E_{6}=-77.0274 .
\end{aligned}
$$

And the fractal dimension is

$$
D_{L}=j+\frac{1}{\left|L E_{j+1}\right|} \sum_{i=1}^{j} L E_{i}=3+\frac{L E_{1}+L E_{2}+L E_{3}}{\left|L E_{4}\right|}=3.292777
$$

Both Lyapunov exponents and fractal dimension indicate that the system (5) is chaotic with the aforementioned set of parameters.
2.2.5. Poincaré Map. As an important analysis technique, the Poincaré map can reflect bifurcation and folding properties of hyperchaos. We have taken

$$
\begin{aligned}
& \Xi_{1}=\left\{\left(u_{1}, u_{2}, u_{3}, u_{4}, u_{5}, u_{6}\right)^{T} \in R^{6}: u_{1}=0\right\}, \\
& \Xi_{2}=\left\{\left(u_{1}, u_{2}, u_{3}, u_{4}, u_{5}, u_{6}\right)^{T} \in R^{6}: u_{2}=0\right\}, \\
& \Xi_{3}=\left\{\left(u_{1}, u_{2}, u_{3}, u_{4}, u_{5}, u_{6}\right)^{T} \in R^{6}: u_{3}=0\right\}, \\
& \Xi_{4}=\left\{\left(u_{1}, u_{2}, u_{3}, u_{4}, u_{5}, u_{6}\right)^{T} \in R^{6}: u_{4}+u_{5}+u_{6}=0\right\}
\end{aligned}
$$

as cross sections and the system parameters are set as $a=10$, $b=40$, and $c=14.9$. Figure 5 shows projections of the Poincaré map on $\left(u_{1}, u_{2}, u_{3}\right)$ space and $\left(u_{1}, u_{2}\right),\left(u_{1}, u_{3}\right)$, and $\left(u_{2}, u_{3}\right)$ planes. From Figure 5 , one can see that the Poincaré map here consists of several limbs with various bifurcations in different directions, which indicates that the system has extremely rich dynamics. Also, the Poincaré maps show that the branches are jointed and united as a single attractor. This proves the existence of the four-wing hyperchaotic attractor of complex Dadras system (5) in Figure 3.

\section{CMHPS between Hyperchaotic and Chaotic Complex Systems with Complex Parameters and with Different Dimensions}

3.1. Scheme of CMHPS. First, we consider a class of $m$-dimensional complex chaotic (hyperchaotic) drive system as

$$
\dot{x}(t)=A x+F(x)
$$

and $n$-dimensional response system with the controller as

$$
\dot{y}(t)=B y+G(y)+P(x, y),
$$

where $x=x^{r}+j x^{i} \in \mathbb{C}^{m}$ and $y=y^{r}+j y^{i} \in \mathbb{C}^{n}$ are complex states vectors, $A \in \mathbb{C}^{m \times m}$ and $B \in \mathbb{C}^{n \times n}$ are the coefficient matrices of $x$ and $y$, while $F$ and $G$ are the nonlinear parts, respectively; $P=\left(p_{1}, p_{2}, \ldots, p_{n}\right)^{T}$ is the controller to be designed.

Next, we consider the definition of CMHPS with a complex transformation matrix of complex systems with complex parameters based on that of CMPS of complex systems with real parameters [22, 23].

Definition 1. For the drive system (16) and response system (17), it is said to be CMHPS with $\Lambda$ between $x(t)$ and $y(t)$ if there exists a norm bounded matrix $\Lambda \in \mathbb{C}^{n \times m}$, such that

$$
\lim _{t \rightarrow+\infty}\|y(t)-\Lambda x(t)\|=0,
$$

while the matrix $\Lambda$ is defined as complex transformation matrix of the drive system (16).

If the error of CMHPS is defined as

$$
e(t)=y(t)-\Lambda x(t),
$$

the objective of this section is to design a controller $P$ to ensure that synchronization error tends to zero; that is,

$$
\lim _{t \rightarrow+\infty}\|e(t)\|=\lim _{t \rightarrow+\infty}\|y(t)-\Lambda x(t)\|=0 .
$$



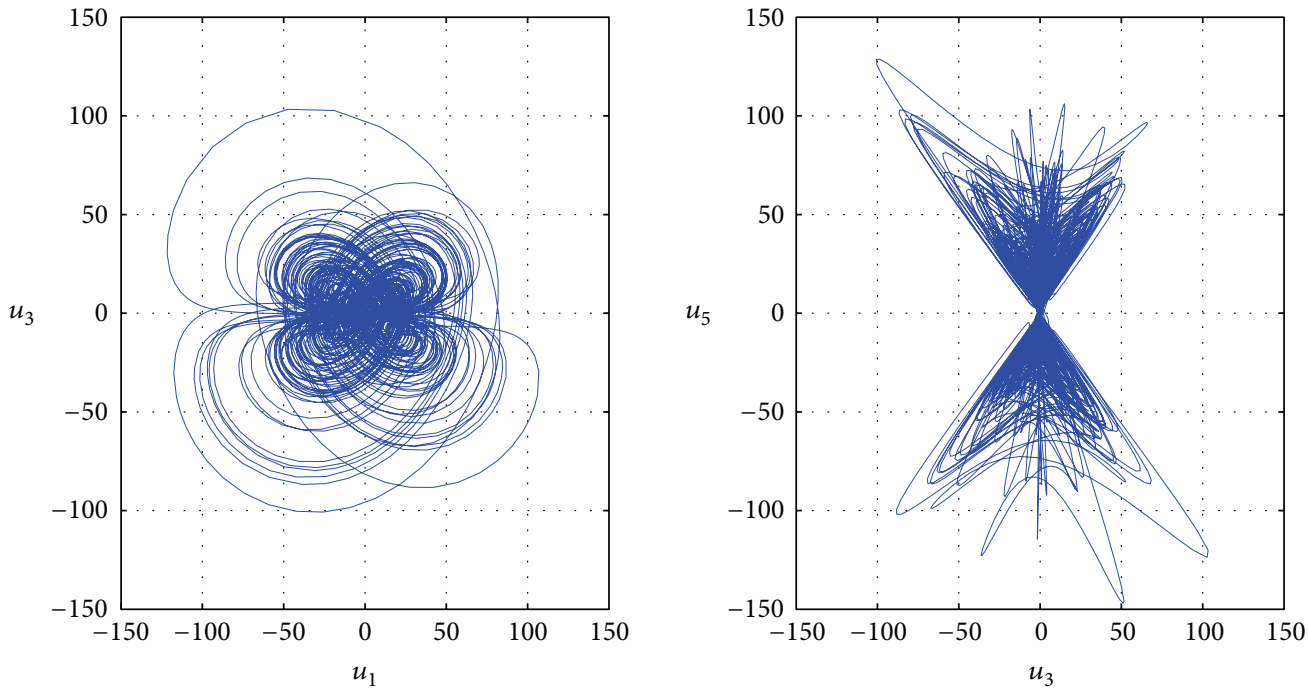

(a) on $\left(u_{1}, u_{3}\right)$ and $\left(u_{3}, u_{5}\right)$ plane
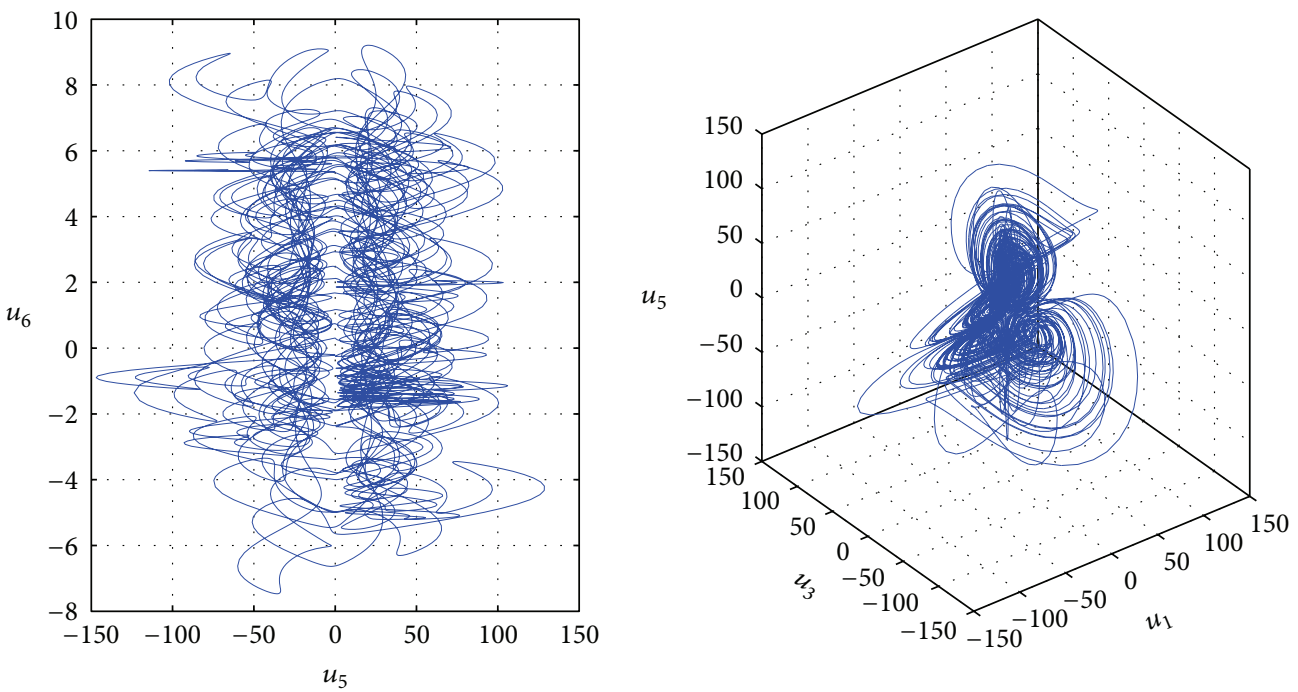

(b) on $\left(u_{5}, u_{6}\right)$ plane and $\left(u_{1}, u_{3}, u_{5}\right)$ space
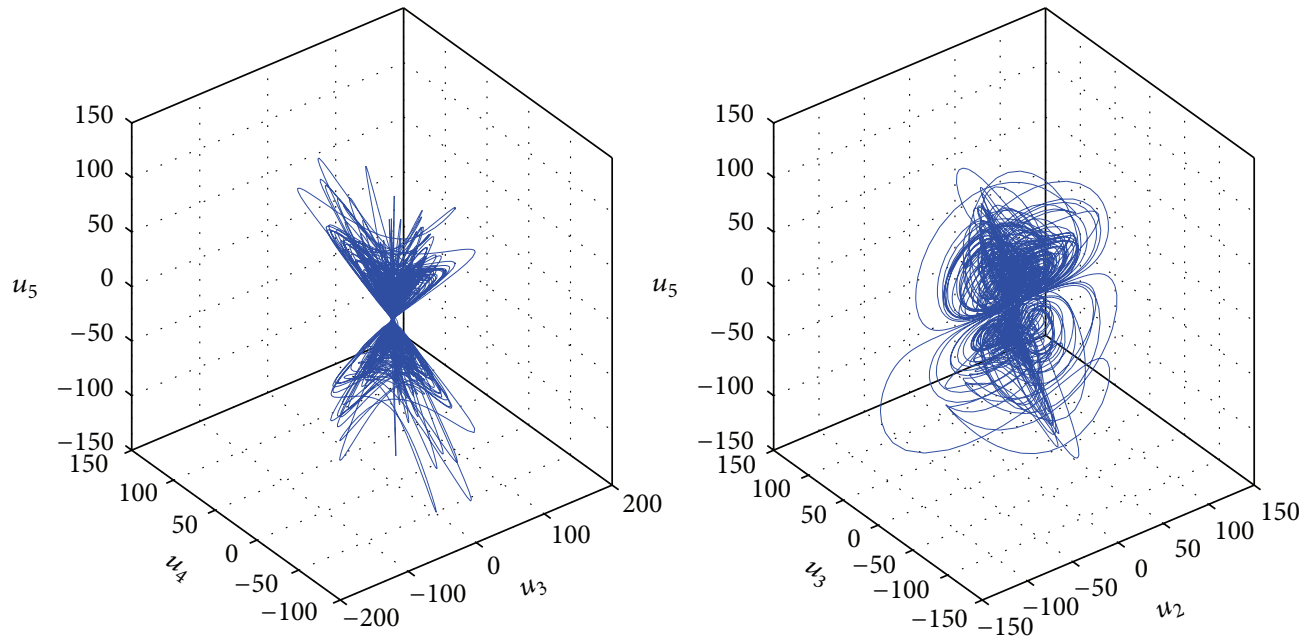

(c) on $\left(u_{3}, u_{4}, u_{5}\right)$ and $\left(u_{2}, u_{3}, u_{5}\right)$ space

FIGURE 3: The hyperchaotic attractors of complex Dadras system (5) with $a=10, b=40$, and $c=14.9$. 

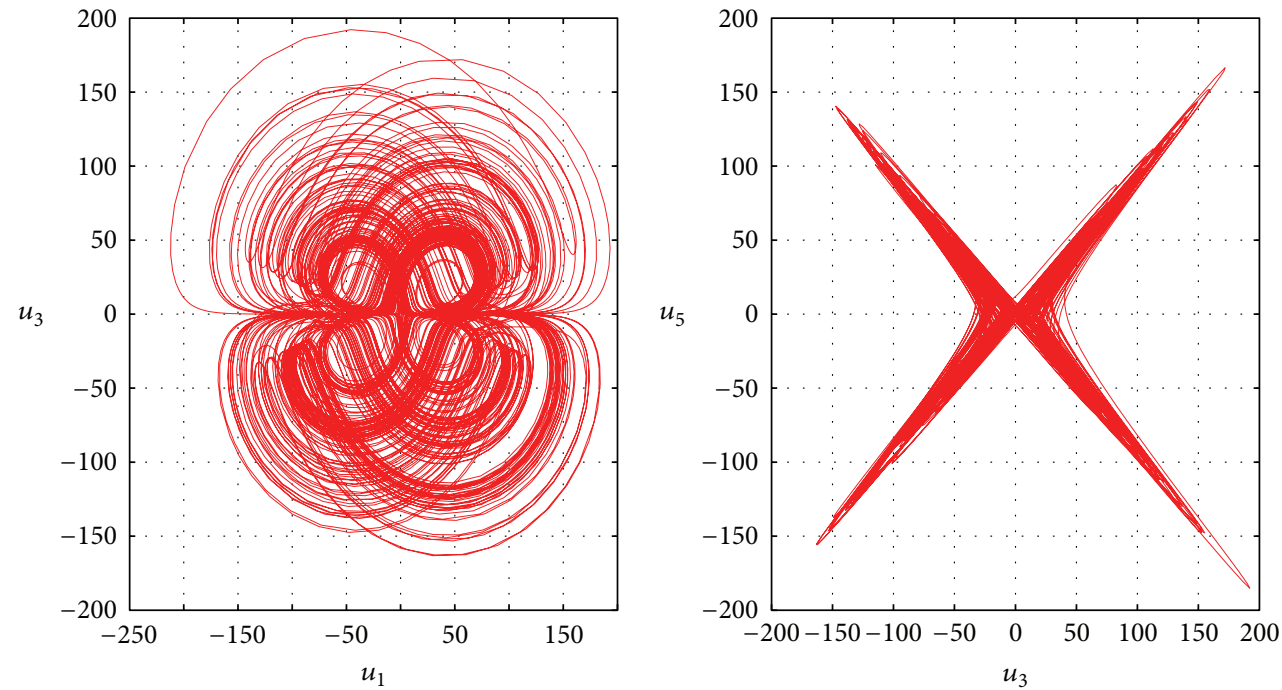

(a) on $\left(u_{1}, u_{3}\right)$ and $\left(u_{3}, u_{5}\right)$ plane
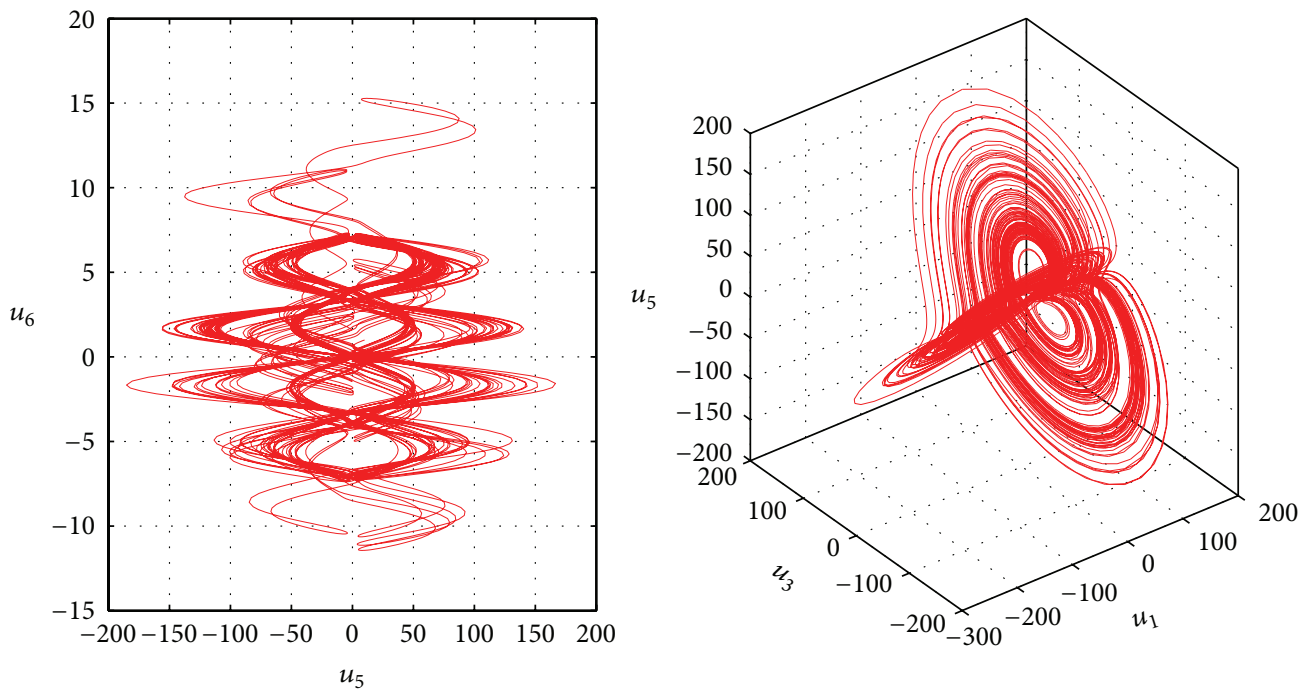

(b) on $\left(u_{5}, u_{6}\right)$ plane and $\left(u_{1}, u_{3}, u_{5}\right)$ space
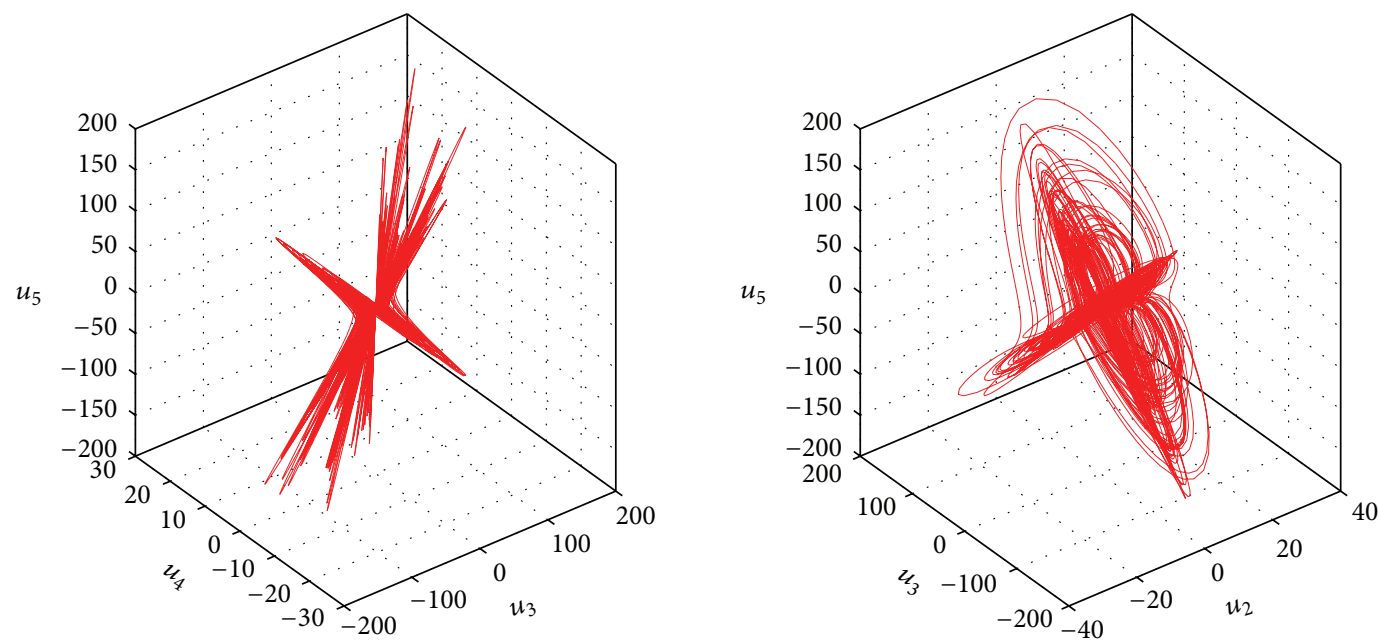

(c) on $\left(u_{3}, u_{4}, u_{5}\right)$ and $\left(u_{2}, u_{3}, u_{5}\right)$ space

Figure 4: The chaotic attractors of complex Dadras system (5) with $a=10, b=40$, and $c=49$. 


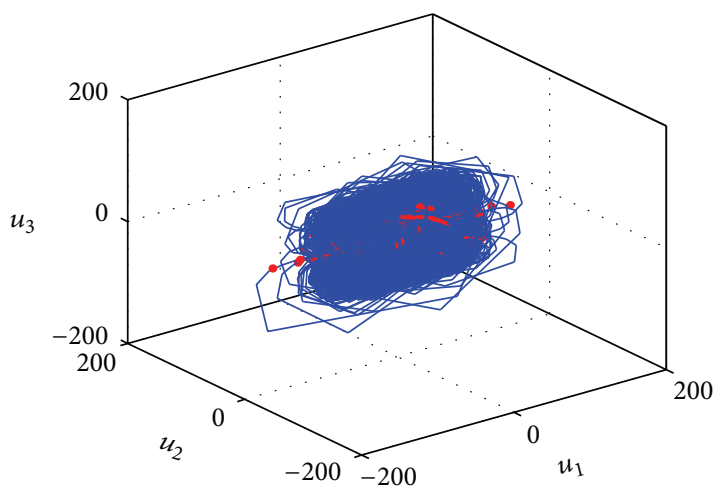

(a)

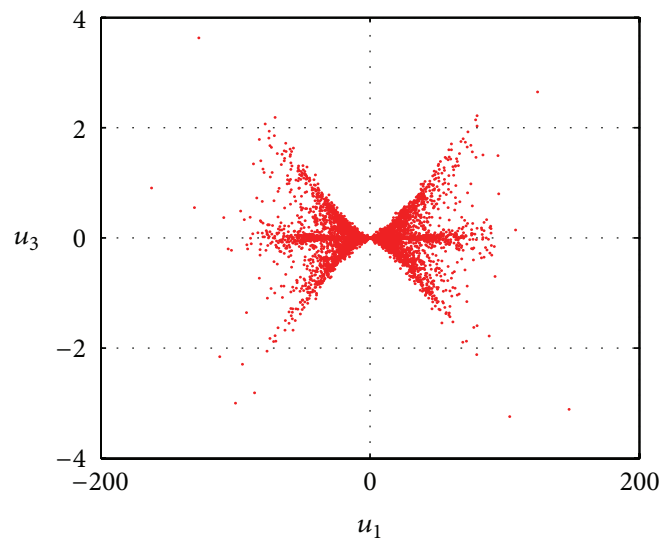

(c)

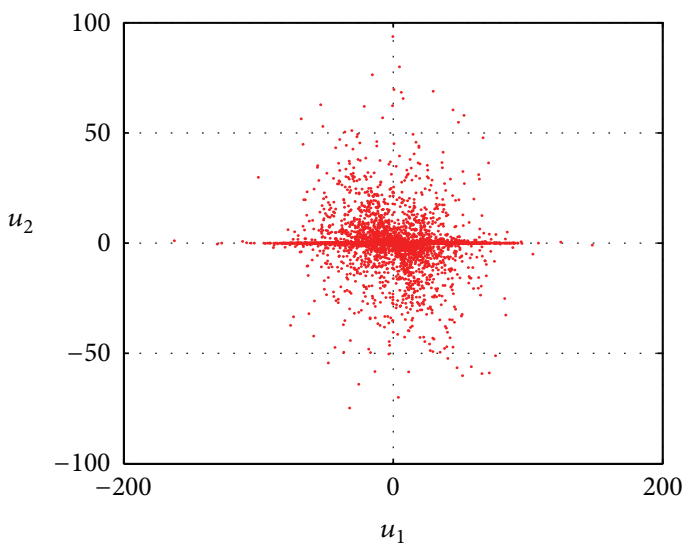

(b)

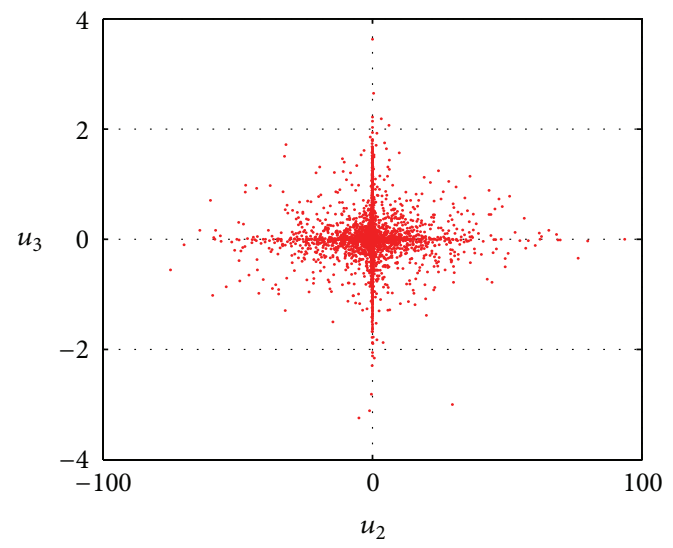

(d)

FIGURE 5: Projections of Poincaré maps of the four-wing hyperchaotic attractors of complex Dadras system (5) with parameters $a=10$, $b=40$, and $c=14.9$, on different crossing sections: $u_{4}+u_{5}+u_{6}=0 ; u_{3}=0 ; u_{2}=0 ; u_{1}=0$.

Remark 2. Most of the classical chaotic (hyperchaotic) complex system can be formed as system (16), such as complex Lorenz system, complex Chen system, complex Lü system, complex hyperchaotic Lorenz system, and complex Duffing system.

Lemma 3. For a matrix $Q \in \mathbb{C}^{n \times n}$, all of the real parts of its eigenvalues are negative; denoted $\operatorname{Re}\left(\lambda_{i}(Q)\right)<0, i=1,2, \ldots$, $n$, then [30]

$$
\lim _{t \rightarrow+\infty} \exp (Q t)=0 .
$$

Theorem 4. For given complex transformation matrix $\Lambda$ and initial conditions $x(0), y(0)$, the complex nonlinear controller is designed as

$$
P=-G(y)+\Lambda(F(x)+A x)-B \Lambda x-K e ;
$$

then, CMHPS between the response system (17) and drive system (16) will occur with desired complex transformation matrix $\Lambda$ asymptotically if $\operatorname{Re}\left(\lambda_{i}(B-K)\right)<0, i=1,2, \ldots, n$, where $K \in \mathbb{C}^{n \times n}$ is the complex control gain matrix.

Proof. Insertion of (16) and (17) into (19) gives

$$
\dot{e}(t)=B e+B \Lambda x+G(y)-\Lambda A x-\Lambda F(x)+P .
$$

Substituting controller (22) into system (23), we can obtain

$$
\dot{e}(t)=(B-K) e(t)
$$

or

$$
\dot{e}(t)-(B-K) e(t)=0 \text {. }
$$

By multiplying $\exp (-(B-K) t)$ on both sides of (25), one has that the left-hand side and the right-hand side of (25) take the form, respectively,

$$
\frac{d(\exp (-(B-K) t) e(t))}{d t}=0 .
$$

Integrating two sides of (26) on time interval $[0, t]$ and with the initial condition $e(0)=\xi$, we can get

$$
e(t)=\exp ((B-K) t) \xi
$$

Then, there exists a suitable $K \in \mathbb{C}^{n \times n}$ such that $\operatorname{Re}\left(\lambda_{i}(B-\right.$ $K))<0, i=1,2, \ldots, n$, to each $B$, and we get the following by Lemma 3:

$$
\lim _{t \rightarrow+\infty} e(t)=\lim _{t \rightarrow+\infty} \exp ((B-K) t) \xi=0 .
$$


Thus, we arrive at CMHPS with desired complex transformation matrix $\Lambda$ between the complex systems (17) and (16) by using the controller (22) asymptotically. The proof is completed.

Remark 5. Note that one can adjust complex transformation matrix $\Lambda$ arbitrarily without worrying about the control robustness, and $K \in \mathbb{C}^{n \times n}$ is the complex control gain matrix. In particular, if $A$ and $B$ are real, Theorem 4 is also applied to achieve CMHPS of complex chaotic systems with real parameters.

Remark 6. In particular, if the dimension of the drive system equals that of the response system, that is, $m=n$, and the transformation matrix $\Lambda$ is diagonal, the CMHPS can be simplified to CFSHPS [21], CMPS [22, 23] CPS, FSHPS [18], MPS, and PS [17] with same dimension.

Remark 7. If the scale matrix $\Lambda=0$, the synchronization problem degenerates to the control of complex chaotic (hyperchaotic) system.

3.2. Realization of Synchronization. Throughout this section, we will verify the effectiveness of the proposed method. Two examples are respectively used to discuss two kinds of cases: increased order synchronization $(m<n)$ and reduced order synchronization $(m>n)$.

3.2.1. CMHPS of Complex Hyperchaotic Dadras Drive System and Complex Chaotic Lorenz Response System with Complex Parameters. In order to observe reduced order CMHPS behaviors, it is assumed that new 4-dimensional hyperchaotic complex Dadras system (5) drives 3-dimensional chaotic complex Lorenz system (2) with complex parameters [4]. Therefore, the drive system reads in the form

$$
\begin{aligned}
& \dot{x}_{1}=a_{1} x_{1}-x_{2} x_{3}+x_{4}, \\
& \dot{x}_{2}=-a_{2} x_{2}+x_{1} x_{3}, \\
& \dot{x}_{3}=\frac{1}{2}\left[\bar{x}_{1}\left(x_{2}+x_{4}\right)+x_{1}\left(\bar{x}_{2}+\bar{x}_{4}\right)\right]-a_{3} x_{3}, \\
& \dot{x}_{4}=-\frac{1}{2}\left(x_{2}+\bar{x}_{2}\right),
\end{aligned}
$$

where

$$
\begin{aligned}
& A=\left[\begin{array}{cccc}
a_{1} & 0 & 0 & 1 \\
0 & -a_{2} & 0 & 0 \\
0 & 0 & -a_{3} & 0 \\
0 & -\frac{1}{2} & 0 & 0
\end{array}\right] \\
& F(x)=\left[\begin{array}{c}
-x_{2} x_{3} \\
x_{1} x_{3} \\
\frac{1}{2}\left[\bar{x}_{1}\left(x_{2}+x_{4}\right)+x_{1}\left(\bar{x}_{2}+\bar{x}_{4}\right)\right] \\
-\frac{1}{2} \bar{x}_{2}
\end{array}\right]
\end{aligned}
$$

and $x_{1}=x_{1}^{r}+j x_{1}^{i}, x_{2}=x_{2}^{r}+j x_{2}^{i}$ are complex state variables and $x_{3}, x_{4}$ are real state variables.

The complex response with the controller writes in the form as

$$
\begin{aligned}
& \dot{y}_{1}=b_{1}\left(y_{2}-y_{1}\right)+p_{1}, \\
& \dot{y}_{2}=b_{2} y_{1}-b_{3} y_{2}-y_{1} y_{3}+p_{2}, \\
& \dot{y}_{3}=\frac{1}{2}\left(\bar{y}_{1} y_{2}+y_{1} \bar{y}_{2}\right)-b_{4} y_{3}+p_{3},
\end{aligned}
$$

where

$$
\begin{gathered}
B=\left[\begin{array}{ccc}
-b_{1} & b_{1} & 0 \\
b_{2} & -b_{3} & 0 \\
0 & 0 & -b_{4}
\end{array}\right], \\
G(y)=\left[\begin{array}{c}
0 \\
-y_{1} y_{3} \\
\frac{1}{2}\left(\bar{y}_{1} y_{2}+y_{1} \bar{y}_{2}\right)
\end{array}\right],
\end{gathered}
$$

and $y_{1}=y_{1}^{r}+j y_{1}^{i}, y_{2}=y_{2}^{r}+j y_{2}^{i}$ are complex state variables and $y_{3}$ is a real state variable; $P=\left(p_{1}, p_{2}, p_{3}\right)^{T}$ is the complex controller to be decided.

Complex transformation matrix $\Lambda$ can be chosen as

$$
\Lambda=\left[\begin{array}{cccc}
-j & 0 & 0 & 0 \\
0 & j & 0 & 0 \\
0 & 0 & 0 & -1
\end{array}\right]
$$

the error system $e(t)=y(t)-\Lambda x(t)$ is obtained as

$$
\begin{aligned}
& e_{1}=y_{1}+j x_{1}, \\
& e_{2}=y_{2}-j x_{2}, \\
& e_{3}=y_{3}+x_{4} .
\end{aligned}
$$

In order to satisfy $\operatorname{Re}\left(\lambda_{i}(B-K)\right)<0, i=1,2,3$, we can chose the complex control gain matrix as

$$
K=\left[\begin{array}{ccc}
-b_{1}-\lambda_{1} & b_{1} & 0 \\
b_{2} & -b_{3}-\lambda_{2} & 0 \\
0 & 0 & -b_{4}-\lambda_{3}
\end{array}\right]
$$

where all of the real parts of $\lambda_{1}, \lambda_{2}, \lambda_{3}$ are negative.

Design the complex state feedback controller according to (22) in Theorem 4 as follows: 


$$
\begin{aligned}
P & =-G(y)+\Lambda(F(x)+A x)-B \Lambda x-K e \\
& =\left(\begin{array}{c}
-j\left(\left(a_{1}+b_{1}\right) x_{1}+b_{1} x_{2}-x_{2} x_{3}+x_{4}\right)+\left(b_{1}+\lambda_{1}\right) e_{1}-b_{1} e_{2} \\
j\left(b_{2} x_{1}+\left(b_{3}-a_{2}\right) x_{2}+x_{1} x_{3}\right)+y_{1} y_{3}-b_{2} e_{1}+\left(b_{3}+\lambda_{2}\right) e_{2} \\
\frac{1}{2}\left(x_{2}+\bar{x}_{2}\right)-b_{4} x_{4}-\frac{1}{2}\left(\bar{y}_{1} y_{2}+y_{1} \bar{y}_{2}\right)+\left(b_{4}+\lambda_{3}\right) e_{3}
\end{array}\right) .
\end{aligned}
$$

The parameters of drive system (29) and response system (31) are $a_{1}=10, a_{2}=40, a_{3}=14.9$, and $b_{1}=5, b_{2}=60+0.2 j$, $b_{3}=1-0.6 j, b_{4}=1.8$, respectively. The initial values are randomly chosen as $x_{0}=(10+j, 10+j, 10,1)^{T}$ and $y_{0}=$ $(2+0.2 j, 15+0.2 j,-5)^{T}$, respectively. All of the eigenvalues of $B-K$ are taken as $\lambda_{1}=-6-5 j, \lambda_{2}=-1-j$, and $\lambda_{3}=-10$. The simulation results are demonstrated in Figure 6, where the blue line presents the states of drive system (29) and the red line presents the states of response system (31). The errors of CMHPS converge asymptotically to zero as in Figure 7, where the red line shows the real parts of the errors and the blue line presents the imaginary parts of the errors.

Hence, the above results show that CMHPS has been achieved between 4-dimensional hyperchaotic complex Dadras system (29) and 3-dimensional complex Lorenz system (31) with complex parameters.

3.2.2. CMHPS of Complex Chaotic Lü Drive System and Complex Hyperchaotic Dadras Response System. In order to observe increased order CMHPS behaviors, it is assumed that the 3-dimensional complex chaotic Lü system [7] drives the new 4-dimensional hyperchaotic complex Dadras system.

The drive system for complex Lü system can be described as

$$
\begin{aligned}
& \dot{x}_{1}=a_{1}\left(x_{2}-x_{1}\right) \\
& \dot{x}_{2}=a_{2} x_{2}-x_{1} x_{3} \\
& \dot{x}_{3}=\frac{1}{2}\left(\bar{x}_{1} x_{2}+x_{1} \bar{x}_{2}\right)-a_{3} x_{3},
\end{aligned}
$$

where

$$
\begin{gathered}
A=\left[\begin{array}{ccc}
-a_{1} & a_{1} & 0 \\
0 & a_{2} & 0 \\
0 & 0 & -a_{3}
\end{array}\right], \\
F(x)=\left[\begin{array}{c}
0 \\
-x_{1} x_{3} \\
\frac{1}{2}\left(\bar{x}_{1} x_{2}+x_{1} \bar{x}_{2}\right)
\end{array}\right],
\end{gathered}
$$

and $x_{1}=x_{1}^{r}+j x_{1}^{i}, x_{2}=x_{2}^{r}+j x_{2}^{i}$ are complex state variables and $x_{3}$ is a real state variable. When $a_{1}=29, a_{2}=21$, and $a_{3}=2$, complex Lü system (37) is chaotic.
The complex response system with the controller writes in the form as

$$
\begin{aligned}
& \dot{y}_{1}=b_{1} y_{1}-y_{2} y_{3}+y_{4}+p_{1}, \\
& \dot{y}_{2}=y_{1} y_{3}-b_{2} y_{2}+p_{2}, \\
& \dot{y}_{3}=\frac{1}{2}\left[\bar{y}_{1}\left(y_{2}+y_{4}\right)+y_{1}\left(\bar{y}_{2}+\bar{y}_{4}\right)\right]-b_{3} y_{3}+p_{3}, \\
& \dot{y}_{4}=-\frac{1}{2}\left(y_{2}+\bar{y}_{2}\right)+p_{4},
\end{aligned}
$$

where

$$
\begin{aligned}
B & =\left[\begin{array}{cccc}
b_{1} & 0 & 0 & 1 \\
0 & -b_{2} & 0 & 0 \\
0 & 0 & -b_{3} & 0 \\
0 & -\frac{1}{2} & 0 & 0
\end{array}\right], \\
G(y) & =\left[\begin{array}{c}
-y_{2} y_{3} \\
y_{1} y_{3} \\
\frac{1}{2}\left[\bar{y}_{1}\left(y_{2}+y_{4}\right)+y_{1}\left(\bar{y}_{2}+\bar{y}_{4}\right)\right] \\
-\frac{1}{2} \bar{y}_{2}
\end{array}\right],
\end{aligned}
$$

and $y_{1}=y_{1}^{r}+j y_{1}^{i}, y_{2}=y_{2}^{r}+j y_{2}^{i}$ are complex state variables and $y_{3}, y_{4}$ are real state variables; $P=\left(p_{1}, p_{2}, p_{3}, p_{4}\right)^{T}$ is the complex controller to be decided.

Complex transformation matrix can be taken as

$$
\Lambda=\left[\begin{array}{ccc}
j & 0 & 0 \\
0 & -j & 0 \\
0 & 0 & 1 \\
0 & 0 & -1
\end{array}\right],
$$

the error system $e(t)=y(t)-\Lambda x(t)$ is obtained as

$$
\begin{aligned}
& e_{1}=y_{1}-j x_{1}, \\
& e_{2}=y_{2}+j x_{2}, \\
& e_{3}=y_{3}-x_{3}, \\
& e_{4}=y_{4}+x_{3} .
\end{aligned}
$$

In order to satisfy $\operatorname{Re}\left(\lambda_{i}(B-K)\right)<0, i=1,2,3,4$, we can chose the complex control gain matrix as

$$
K=\left[\begin{array}{cccc}
b_{1}-\lambda_{1} & 0 & 0 & 1 \\
0 & -b_{2}-\lambda_{2} & 0 & 0 \\
0 & 0 & -b_{3}-\lambda_{3} & 0 \\
0 & -\frac{1}{2} & 0 & -\lambda_{4}
\end{array}\right],
$$

where all of the real parts of $\lambda_{1}, \lambda_{2}, \lambda_{3}, \lambda_{4}$ are negative. 


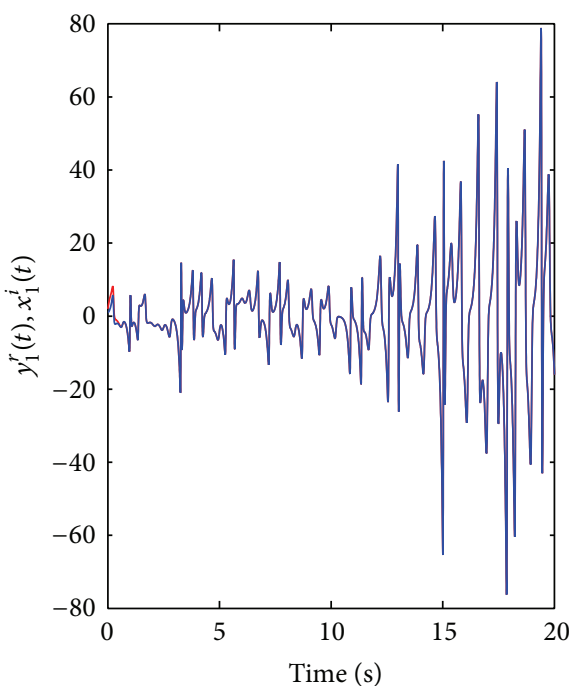

(a) $y_{1}^{r}$ synchronizes $x_{1}^{i}$

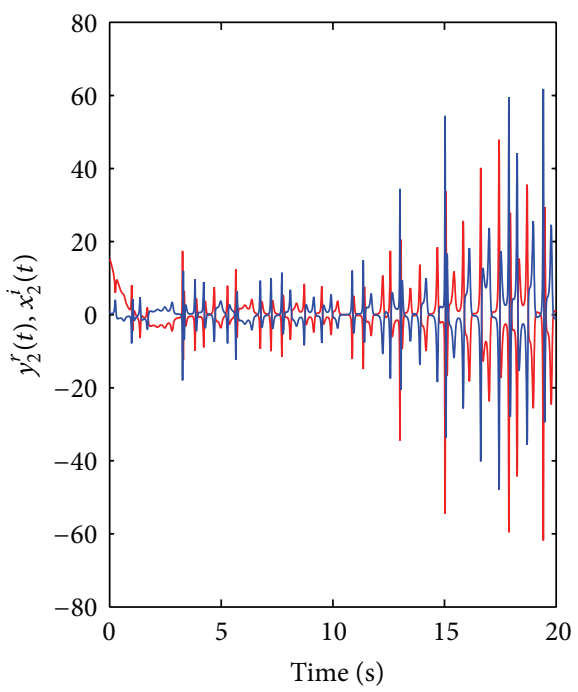

(c) $y_{2}^{r}$ antisynchronizes $x_{2}^{i}$

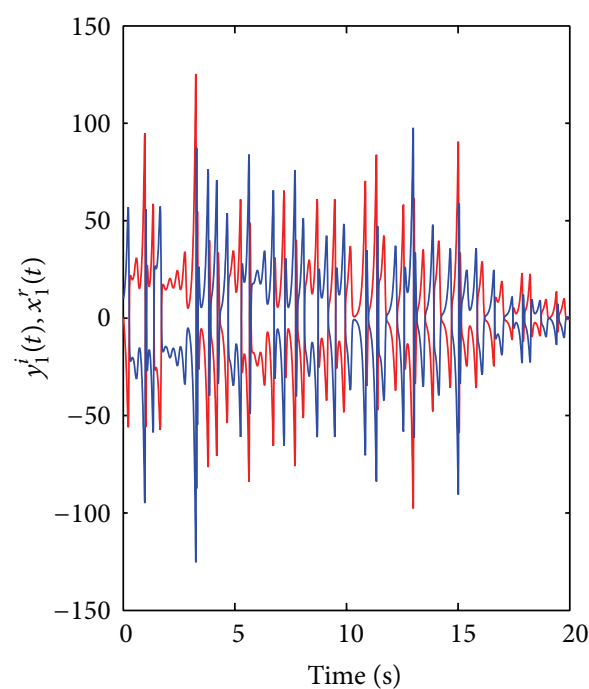

(b) $y_{1}^{i}$ antisynchronizes $x_{1}^{r}$

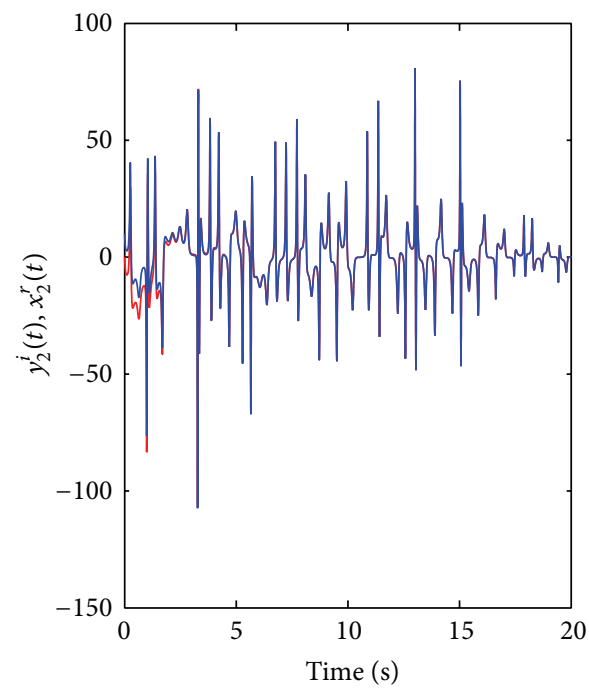

(d) $y_{2}^{i}$ synchronizes $x_{2}^{r}$

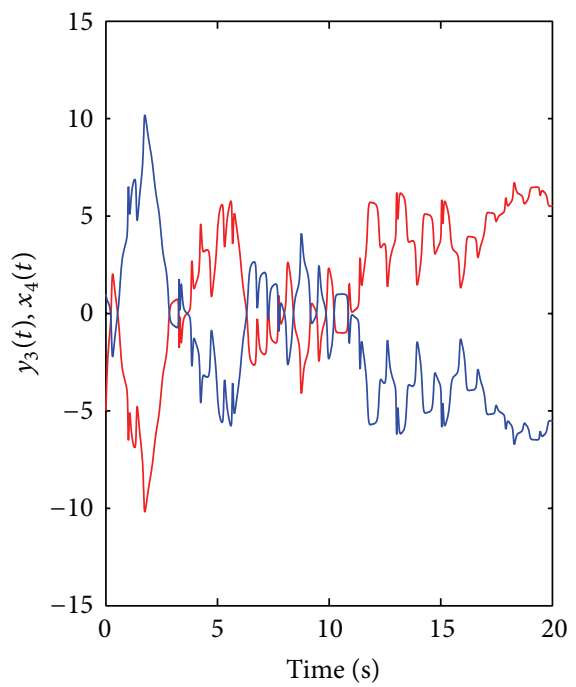

(e) $y_{3}$ antisynchronizes $x_{4}$

FIGURE 6: Reduced order synchronization-CMHPS between 4-dimensional complex hyperchaotic Dadras drive system (29) (blue line) and 3-dimensional complex chaotic Lorenz response system (31) (red line) with the controller (36). 


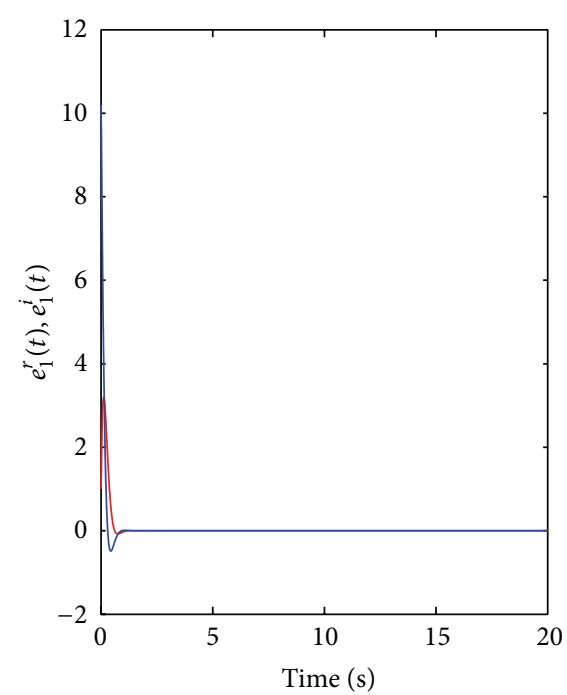

(a) The dynamic of $e_{1}$

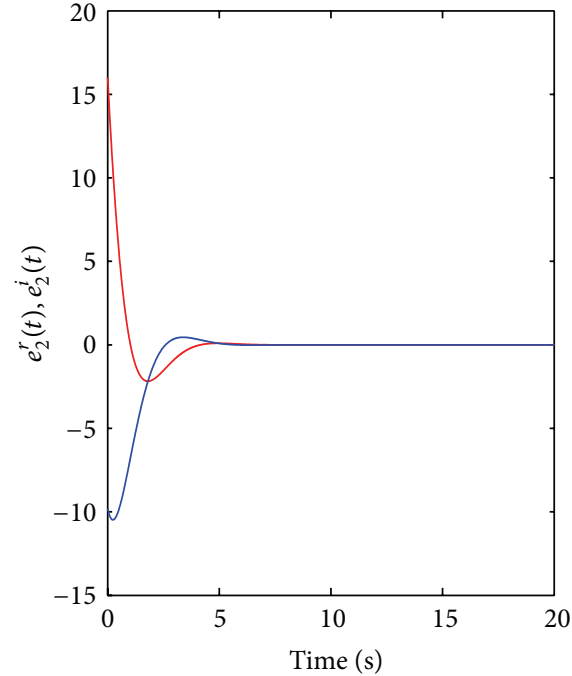

(b) The dynamic of $e_{2}$

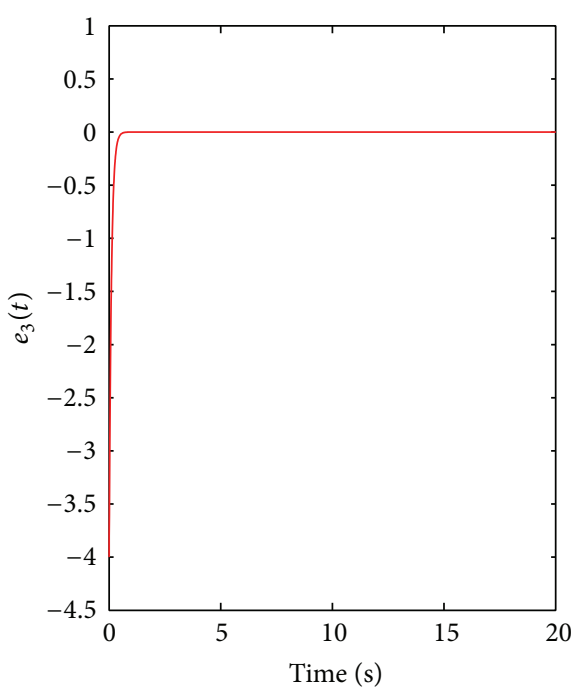

(c) The dynamic of $e_{3}$

FiguRe 7: CMHPS error dynamics between 4-dimensional complex hyperchaotic Dadras drive system (29) and 3-dimensional complex chaotic Lorenz response system (31).

Design the controller according to (22) in Theorem 4 as follows:

$$
\begin{aligned}
P= & -G(y)+\Lambda(F(x)+A x)-B \Lambda x-K e \\
& =\left(\begin{array}{c}
j\left(-\left(a_{1}+b_{1}\right) x_{1}+a_{1} x_{2}\right)+x_{3}+y_{2} y_{3}-\left(b_{1}-\lambda_{1}\right) e_{1}-e_{4} \\
-j\left(\left(a_{2}+b_{2}\right) x_{2}-x_{1} x_{3}\right)-y_{1} y_{3}+\left(b_{2}+\lambda_{2}\right) e_{2} \\
\frac{1}{2}\left(\bar{x}_{1} x_{2}+x_{1} \bar{x}_{2}-\bar{y}_{1}\left(y_{2}+y_{4}\right)-y_{1}\left(\bar{y}_{2}+\bar{y}_{4}\right)\right)+\left(b_{3}-a_{3}\right) x_{3}+\left(b_{3}+\lambda_{3}\right) e_{3} \\
\frac{1}{2}\left(\bar{y}_{2}-j x_{2}-\bar{x}_{1} x_{2}-x_{1} \bar{x}_{2}\right)+a_{3} x_{3}+\frac{1}{2} e_{2}+\lambda_{4} e_{4}
\end{array}\right) .
\end{aligned}
$$

The parameters of drive system (37) and response system (39) are $a_{1}=29, a_{2}=21, a_{3}=2$, and $b_{1}=10, b_{2}=40$, $b_{3}=14.9$, respectively. The initial values are randomly chosen as $x_{0}=(4-2 j, 3+j,-10)^{T}$ and $y_{0}=(10+j, 10+j, 10,1)^{T}$, 


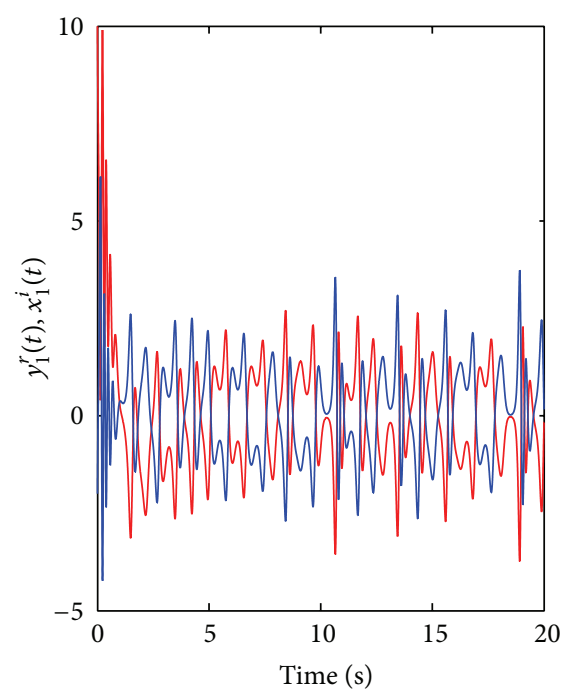

(a) $y_{1}^{r}$ antisynchronizes $x_{1}^{i}$

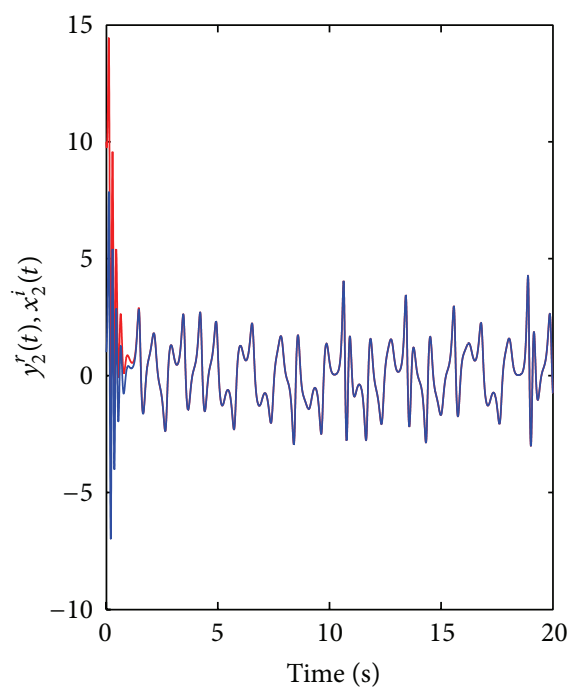

(c) $y_{2}^{r}$ synchronizes $x_{2}^{i}$

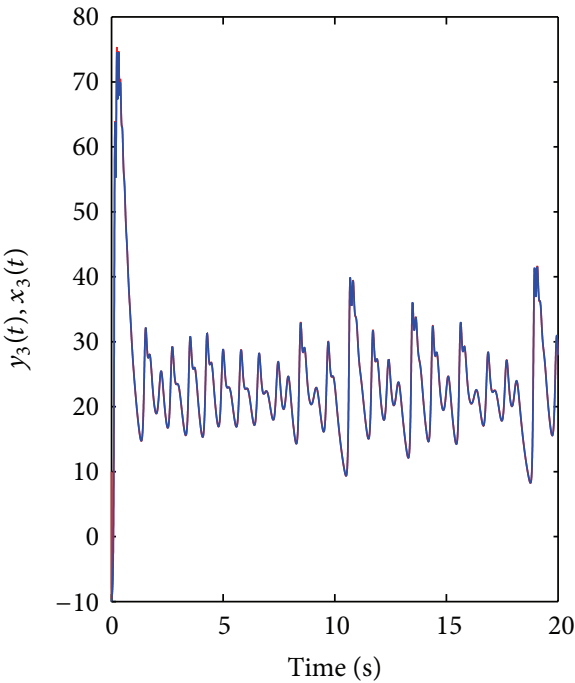

(e) $y_{3}$ synchronizes $x_{3}$

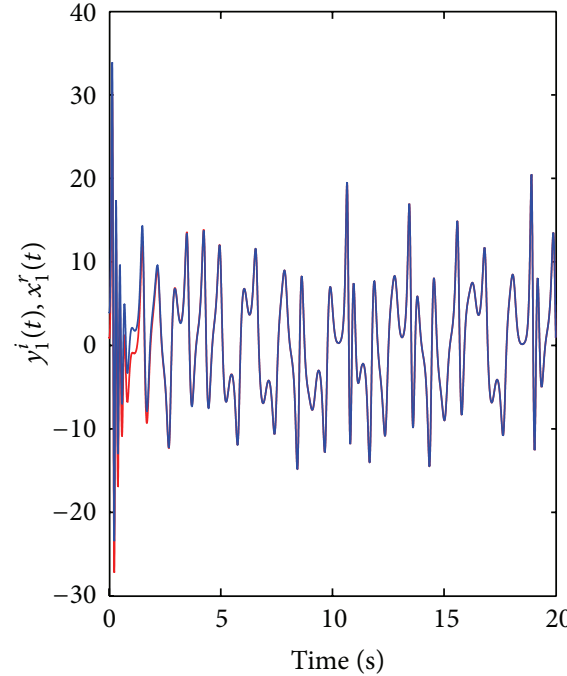

(b) $y_{1}^{i}$ synchronizes $x_{1}^{r}$

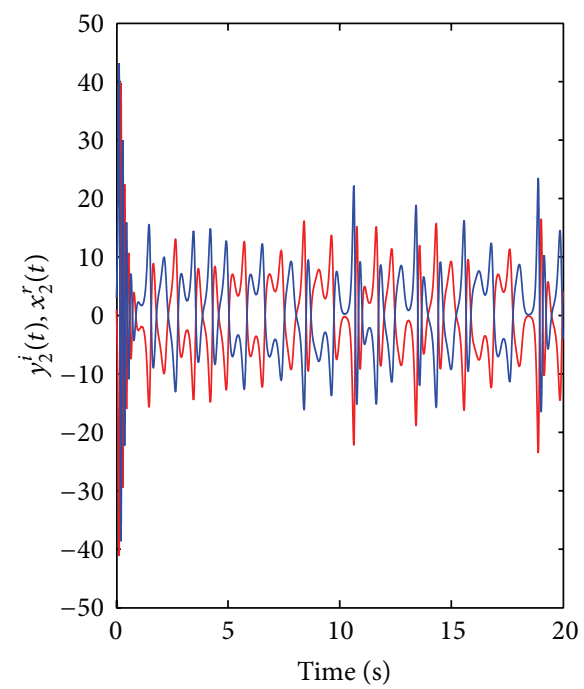

(d) $y_{2}^{i}$ antisynchronizes $x_{2}^{r}$

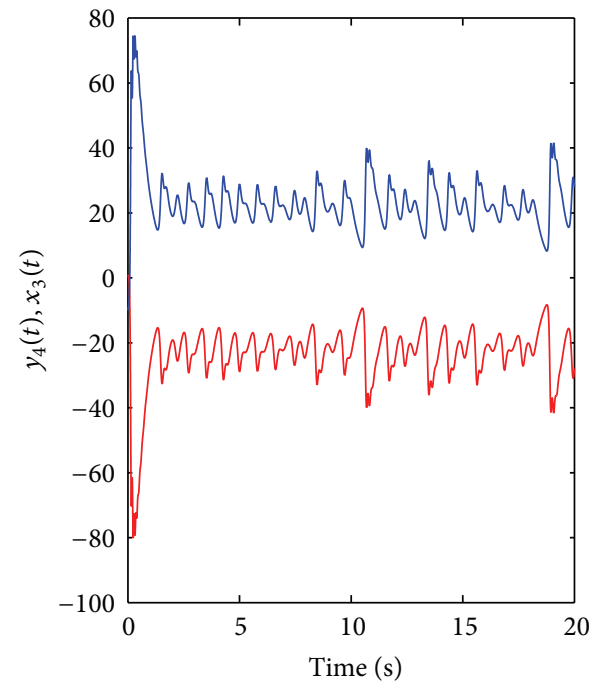

(f) $y_{4}$ antisynchronizes $x_{4}$

FIGURE 8: Increased order synchronization-CMHPS between 3-dimensional complex chaotic Lü drive system (37) (blue line) and 4dimensional complex hyperchaotic Dadras response system (39) (red line) with the controller (44). 


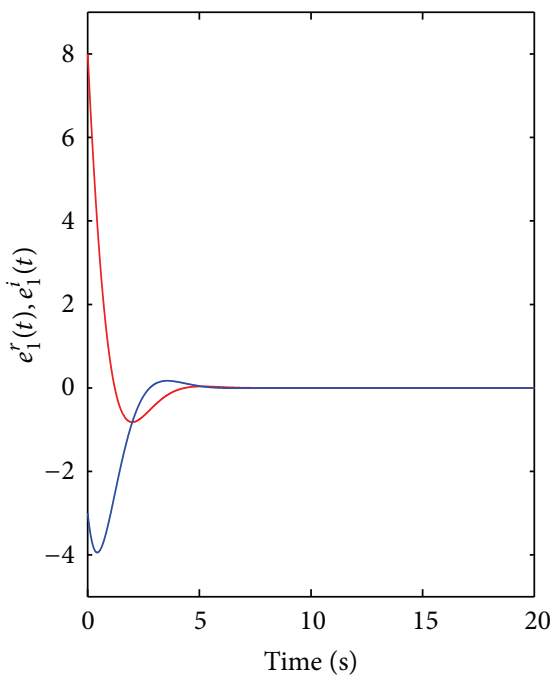

(a) The dynamic of $e_{1}$

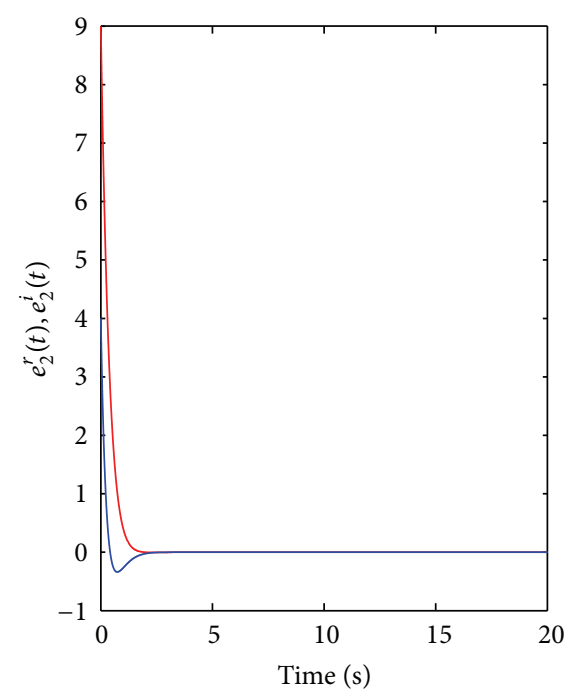

(b) The dynamic of $e_{2}$

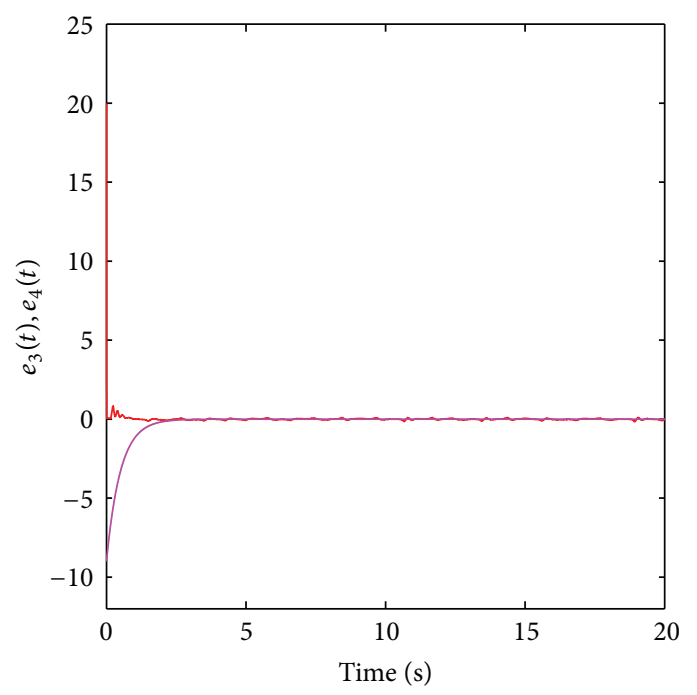

(c) The dynamics of $e_{3}$ (red) and $e_{4}$ (pink)

FIGURE 9: CMHPS error dynamics between 3-dimensional complex chaotic Lü drive system (37) and 4-dimensional complex hyperchaotic Dadras response system (39).

respectively. All of the eigenvalues of $B-K$ are taken as $\lambda_{1}=$ $-1-j, \lambda_{2}=-3-j, \lambda_{3}=-500$, and $\lambda_{4}=-2$. The simulation results are demonstrated in Figure 8, where the blue line presents the states of drive system (37) and the red line presents the states of response system (39). The errors of CMHPS converge asymptotically to zero as in Figure 9, where the red line shows the real parts of the errors and the blue line presents the imaginary parts of the errors.

Hence, the above results show that CMHPS has been achieved between complex chaotic Lü drive system (37) and complex hyperchaotic Dadras response system (39).

\section{Conclusion}

In this work, a new Dadras system with complex variables is introduced. Some dynamic properties of the new system are described including Lyapunov exponents, fractal dimensions, and Poincaré maps, and both four-wing hyperchaotic and chaotic attractors are shown.

More importantly, a general scheme of CMHPS is addressed for different dimensional hyperchaotic and chaotic complex systems with complex parameters, where the drive and response systems could be asymptotically synchronized up to a desired complex transformation matrix, not a diagonal matrix. Complex nonlinear controller and complex gain matrix are introduced to control the response system to become a projection of the drive system.

Furthermore, CMHPS between 4-dimensional complex hyperchaotic Dadras drive system and 3-dimensional complex chaotic Lorenz response system with complex parameters is implemented as an example to discuss reduced order synchronization, and CMHPS between 3-dimensional 
complex chaotic drive Lü system and 4-dimensional complex hyperchaotic Dadras response system is implemented as an example to discuss increased order synchronization as well. Numerical results illustrated the effectiveness of the proposed scheme. These theoretical and numerical results provide a bridge between hyperchaotic and chaotic complex systems with complex parameters and with different dimensions.

Finally, it is hoped that the results reported here will be applied in engineering fields such as communication, biology, and medical fields.

\section{Conflict of Interests}

The authors declare that there is no conflict of interests regarding the publication of this paper.

\section{Acknowledgments}

This research was partially supported by the National Nature Science Foundation of China (Grant nos. 61273088, 10971120, and 61001099), the Nature Science Foundation of Shandong Province, China (Grant nos. ZR2010FM010 and ZR2011AL007), and the Shandong Overseas Research and Training Program for University Prominent Young and Middle-Aged Teachers. The authors would like to thank the editors and the reviewers for their constructive comments and suggestions which improved the quality of the paper.

\section{References}

[1] E. N. Lorenz, "Deterministic nonperiodic flow," Journal of the Atmospheric Sciences, vol. 20, pp. 130-141, 1963.

[2] D. J. Tritton, Physical Fluid Dynamics, Clarendon Press, 1988.

[3] J. D. Gibbon and M. J. McGuinness, "The real and complex Lorenz equations in rotating fluids and lasers," Physica D. Nonlinear Phenomena, vol. 5, no. 1, pp. 108-122, 1982.

[4] A. C. Fowler, M. J. McGuinness, and J. D. Gibbon, “The complex Lorenz equations," Physica D. Nonlinear Phenomena, vol. 4, no. 2, pp. 139-163, 1981/82.

[5] A. Rauh, L. Hannibal, and N. B. Abraham, "Global stability properties of the complex Lorenz model," Physica D. Nonlinear Phenomena, vol. 99, no. 1, pp. 45-58, 1996.

[6] G. M. Mahmoud, M. A. Al-Kashif, and S. A. Aly, "Basic properties and chaotic synchronization of complex Lorenz system," International Journal of Modern Physics C, vol. 18, no. 2, pp. 253-265, 2007.

[7] G. M. Mahmoud, T. Bountis, and E. E. Mahmoud, "Active control and global synchronization of the complex Chen and Lü systems," International Journal of Bifurcation and Chaos in Applied Sciences and Engineering, vol. 17, no. 12, pp. 4295-4308, 2007.

[8] A. H. Nayfeh and D. T. Mook, Nonlinear Oscillations, Wiley, New York, NY, USA, 1979.

[9] A. C. Newell and J. V. Moloney, Nonlinear Optics, AddisonWesley, Reading, Mass, USA, 1992.

[10] V. A. Rozhanskii and L. D. Tsendin, Transport Phenomena in Partially Ionized Plasma, Taylor and Francis, London, UK, 2001.

[11] L. Cveticanin, "Resonant vibrations of nonlinear rotors," Mechanism and Machine Theory, vol. 30, no. 4, pp. 581-588, 1995.
[12] R. Dilao and R. Alves-Pires, Nonlinear Dynamics in Particle Accelerators, World Scientific, Singapore, 1996.

[13] G. M. Mahmoud, "Approximate solutions of a class of complex nonlinear dynamical systems," Physica A. Statistical Mechanics and Its Applications, vol. 253, no. 1, pp. 211-222, 1998.

[14] L. Cveticanin, "Analytic approach for the solution of the complex-valued strong non-linear differential equation of Duffing type," Physica A. Statistical Mechanics and Its Applications, vol. 297, no. 3-4, pp. 348-360, 2001.

[15] G. M. Mahmoud, A. A. Mohamed, and S. A. Aly, "Strange attractors and chaos control in periodically forced complex Duffing's oscillators," Physica A. Statistical Mechanics and Its Applications, vol. 292, no. 1-4, pp. 193-206, 2001.

[16] G. M. Mahmoud and T. Bountis, "The dynamics of systems of complex nonlinear oscillators: a review," International Journal of Bifurcation and Chaos in Applied Sciences and Engineering, vol. 14, no. 11, pp. 3821-3846, 2004.

[17] G. M. Mahmoud and E. E. Mahmoud, "Synchronization and control of hyperchaotic complex Lorenz system," Mathematics and Computers in Simulation, vol. 80, no. 12, pp. 2286-2296, 2010.

[18] P. Liu and S. T. Liu, "Robust adaptive full state hybrid synchronization of chaotic complex systems with unknown parameters and external disturbances," Nonlinear Dynamics, vol. 70, no. 1, pp. 585-599, 2012.

[19] M. F. Hu, Z. Y. Xu, and R. Zhang, "Full state hybrid projective synchronization in continuous-time chaotic (hyperchaotic) systems," Communications in Nonlinear Science and Numerical Simulation, vol. 13, no. 2, pp. 456-464, 2008.

[20] Y. Chu, Y. X. Chang, J. G. Zhang, X. F. Li, and X. L. An, "Full state hybrid projective synchronization in hyperchaotic systems," Chaos, Solitons and Fractals, vol. 42, no. 3, pp. 1502-1510, 2009.

[21] F. F. Zhang and S. T. Liu, "Full state hybrid projective synchronization and parameters identification for uncertain chaotic (hyperchaotic) complex systems," Journal of Computational and Nonlinear Dynamics, vol. 9, Article ID 021009, 2014.

[22] F. F. Zhang, S. T. Liu, and W. Y. Yu, "Modified projective synchronization with complex scaling factors of uncertain real chaos and complex chaos," Chinese Physics B, vol. 22, Article ID 120505, 2013.

[23] G. M. Mahmoud and E. E. Mahmoud, "Complex modified projective synchronization of two chaotic complex nonlinear systems," Nonlinear Dynamics, vol. 73, no. 4, pp. 2231-2240, 2013.

[24] Z. G. Li and D. L. Xu, "A secure communication scheme using projective chaos synchronization," Chaos, Solitons and Fractals, vol. 22, no. 2, pp. 477-481, 2004.

[25] Z. Y. Wu, G. R. Chen, and X. C. Fu, "Synchronization of a network coupled with complex-variable chaotic systems," Chaos, vol. 22, no. 2, Article ID 023127, 2012.

[26] Y. Zhang and J. J. Jiang, "Nonlinear dynamic mechanism of vocal tremor from voice analysis and model simulations," Journal of Sound and Vibration, vol. 316, no. 1-5, pp. 248-262, 2008.

[27] G. M. Mahmoud, E. E. Mahmoud, and A. A. Arafa, "On projective synchronization of hyperchaotic complex nonlinear systems based on passive theory for secure communications," Physica Scripta, vol. 87, no. 5, Article ID 055002, 2013.

[28] S. Dadras, H. R. Momeni, G. Qi, and Z.-l. Wang, "Four-wing hyperchaotic attractor generated from a new $4 \mathrm{D}$ system with one equilibrium and its fractional-order form," Nonlinear Dynamics, vol. 67, no. 2, pp. 1161-1173, 2012. 
[29] A. Wolf, J. B. Swift, H. L. Swinney, and J. A. Vastano, "Determining Lyapunov exponents from a time series," Physica D. Nonlinear Phenomena, vol. 16, no. 3, pp. 285-317, 1985.

[30] H. C. Wei and X. C. Zheng, The Matrix Theory in Engineering, China University of Petroleum Press, Dongying, China, 1999. 


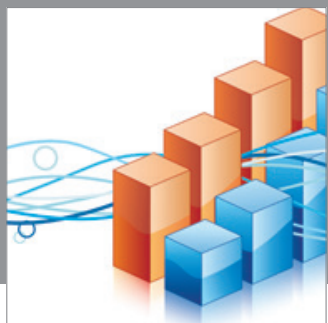

Advances in

Operations Research

mansans

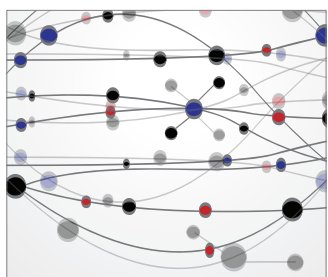

The Scientific World Journal
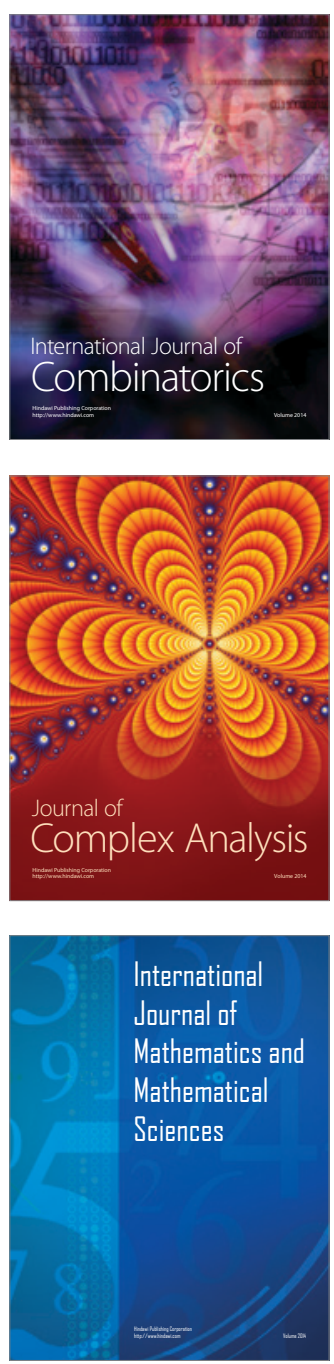
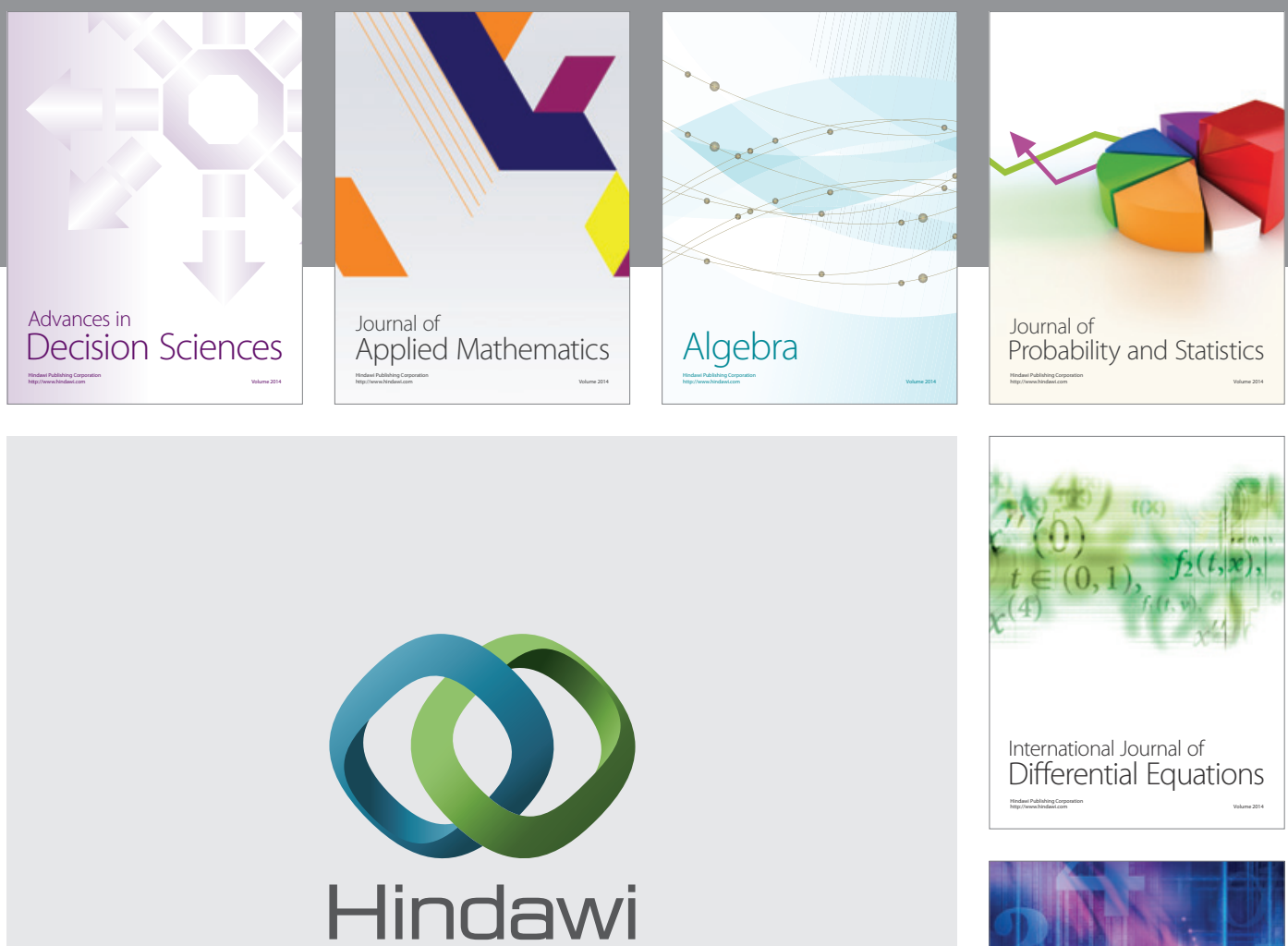

Submit your manuscripts at http://www.hindawi.com
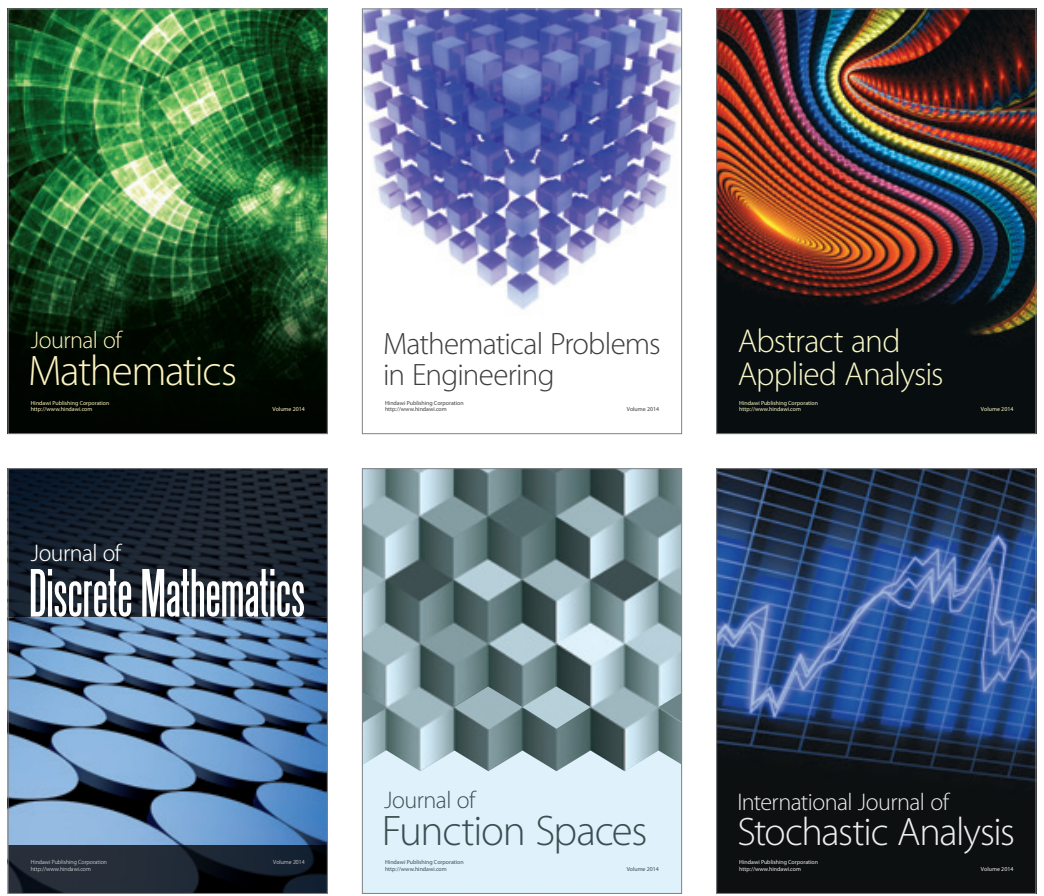

Journal of

Function Spaces

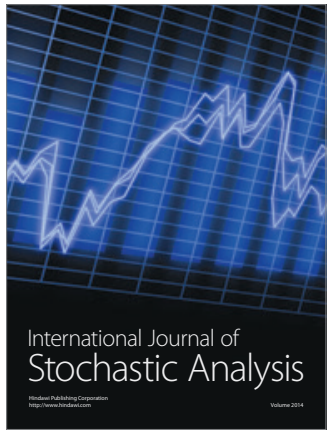

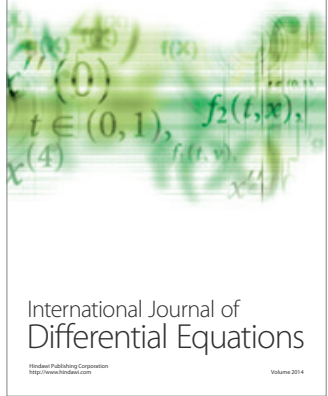
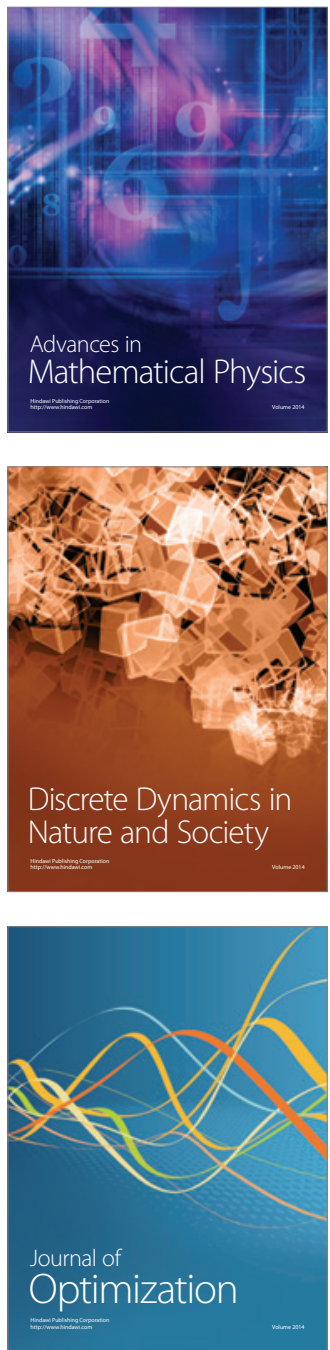